\title{
Operated semigroups, Motzkin paths and rooted trees
}

\author{
Li Guo
}

Received: 27 April 2007 / Accepted: 20 December 2007 / Published online: 10 January 2008

(C) Springer Science+Business Media, LLC 2008

\begin{abstract}
Combinatorial objects such as rooted trees that carry a recursive structure have found important applications recently in both mathematics and physics. We put such structures in an algebraic framework of operated semigroups. This framework provides the concept of operated semigroups with intuitive and convenient combinatorial descriptions, and at the same time endows the familiar combinatorial objects with a precise algebraic interpretation. As an application, we obtain constructions of free Rota-Baxter algebras in terms of Motzkin paths and rooted trees.
\end{abstract}

Keywords Operated semigroups $\cdot$ Operated algebras $\cdot$ Planar rooted trees $\cdot$ Motzkin paths $\cdot$ Dyck paths $\cdot$ Rota-Baxter algebras

\section{Introduction}

\subsection{Motivation}

This paper explores the relationship between two subjects that have been studied separately until recently. One subject considers algebraic structures, such as semigroups and associative algebras, with an operator acting on them. Such structures include differential algebras, difference algebras and Rota-Baxter algebras (See Example 1.3 for the definitions). Another subject studies objects, often combinatorial in nature, that have underlying recursive structures, such as rooted trees and Motzkin paths. Since the 1990s, algebraic structures on rooted trees have been studied in the work of Connes and Kreimer [10] on the renormalization of quantum field theory, Grossman and Larson [23] on data structures and of Loday and Ronco [34] on operands. The grafting operator on trees plays an important role in their works. More recently [2,

\footnotetext{
L. Guo $(\bowtie)$

Department of Mathematics and Computer Science, Rutgers University, Newark, NJ 07102, USA

e-mail: liguo@rutgers.edu
} 
16], free Rota-Baxter algebras were constructed using planar rooted trees with special decorations.

In this paper, we relate these two subjects through the concepts of operated semigroups, operated monoids and operated algebras. We establish that free operated semigroups have natural combinatorial interpretation in terms of Motzkin paths and planar rooted forests. This freeness characterization of rooted forests and Motzkin paths gives an algebraic explanation of the fundamental roles played by these combinatorial objects and their related numerical sequences such as the Catalan numbers and Motzkin numbers [42]. This characterization should be useful in further algebraic studies of these combinatorial objects. This connection also endows the concept of operated algebras and semigroups with familiar combinatorial contents, giving significance to these operated algebraic structures beyond the abstract generalization. As a consequence, we obtain several constructions of free Rota-Baxter algebras which can be adopted to free objects in the other related algebraic structures.

\subsection{Definitions and examples}

Definition 1.1 An operated semigroup (or a semigroup with an operator) is a semigroup $U$ together with an operator $\alpha: U \rightarrow U . \alpha$ is called the distinguished operator on $U$. A morphism from an operated semigroup $(U, \alpha)$ to an operated semigroup $(V, \beta)$ is a semigroup homomorphism $f: U \rightarrow V$ such that $f \circ \alpha=\beta \circ f$.

More generally, let $\Omega$ be a set. An $\Omega$-operated semigroup is a semigroup $U$ together with a set of operators $\alpha_{\omega}: U \rightarrow U, \omega \in \Omega$. In other words, an $\Omega$ operated semigroup is a pair $(U, \alpha)$ with a semigroup $U$ and a map $\alpha: \Omega \rightarrow$ $\operatorname{Map}(U, U), \alpha(\omega)=\alpha_{\omega}$. Here $\operatorname{Map}(U, U)$ is the set of maps from $U$ to $U$. A morphism from an $\Omega$-operated semigroup $\left(U,\left\{\alpha_{\omega}, \omega \in \Omega\right\}\right)$ to an $\Omega$-operated semigroup $\left(V,\left\{\beta_{\omega}, \omega \in \Omega\right\}\right)$ is a semigroup homomorphism $f: U \rightarrow V$ such that $f \circ \alpha_{\omega}=$ $\beta_{\omega} \circ f$ for $\omega \in \Omega$.

Remark 1.2 When a semigroup is replaced by a monoid we obtain the concept of an $(\Omega-)$ operated monoid. Let $\mathbf{k}$ be a commutative ring. We similarly define the concepts of an $(\Omega$-)operated k-algebra or $(\Omega$-)operated nonunitary k-algebra.

Example 1.3 Here are some examples of operated semigroups and $\mathbf{k}$-algebras.

(a) A semigroup is an operated semigroup when the distinguished operator is taken to be the identity;

(b) A differential algebra $[33,41]$ is an algebra $A$ with a linear operator $d: A \rightarrow A$ such that

$$
d(x y)=d(x) y+x d(y), \forall x, y \in A ;
$$

(c) A difference algebra [9] is an algebra $A$ with an algebra endomorphism on $A$;

(d) Let $\lambda$ be fixed in the ground ring k. A Rota-Baxter algebra (of weight $\lambda$ ) [4, 7, $15,26,36,37]$ is defined to be an associative algebra $A$ with a linear operator $P$ such that

$$
P(x) P(y)=P(x P(y))+P(P(x) y)+\lambda P(x y), \forall x, y \in A
$$


(e) A differential algebras of weight $\lambda$ [28] is defined to be an algebra $A$ together with a linear operator $d: A \rightarrow A$ such that

$$
d(x y)=d(x) y+x d(y)+\lambda d(x) d(y), \forall x, y \in A .
$$

Other examples of operated algebras can be found in [37].

Here are some $\Omega$-operated algebras with multiple operators.

(a) A $\Delta$-differential algebras [33] is an algebra with multiple differential operators $\delta \in \Delta$ that commute with each other;

(b) A $\sigma \delta$-algebra [40] is an algebra with a commuting pair of a difference operator $\sigma$ and a differential operator $\delta$;

(c) A differential Rota-Baxter algebra of weight $\lambda$ [28] is an algebra with a differential operator $d$ of weight $\lambda$ and a Rota-Baxter operator $P$ of weight $\lambda$, such that $d \circ P=\mathrm{i} d$. This last relation is a natural generalization of the First Fundamental Theorem of Calculus when $d$ is taken to be the usual derivation and $P$ is the integral operator $P[f](x)=\int_{a}^{x} f(t) d t$;

(d) As a variation, we can consider an algebra with a differential operator and a RotaBaxter operator of weight -1 that commute with each other. It arises naturally in the study of multiple zeta values by renormalization methods [30, 31];

(e) A Rota-Baxter family on an algebra $R$ is a collection of linear operators $P_{\omega}$ on $R$ with $\omega$ in a semigroup $\Omega$, such that

$$
P_{\alpha}(x) P_{\beta}(y)=P_{\alpha \beta}\left(P_{\alpha}(x) y\right)+P_{\alpha \beta}\left(x P_{\beta}(y)\right)+\lambda P_{\alpha \beta}(x y), \forall x, y \in A, \alpha, \beta \in \Omega .
$$

It arises naturally in renormalization of quantum field theory [14, Prop. 9.1].

Our main goal here is to give combinatorial constructions of free objects in the category of $\Omega$-operated semigroups and $\Omega$-operated monoids. They naturally give free objects in the category of operated algebras. These free objects are obtained as the adjoint functors of the forgetful functors from the category of operated semigroups and operated monoids to the category of sets in the usual way. More precisely,

Definition 1.4 A free operated semigroup on a set $X$ is an operated semigroup $\left(U_{X}, \alpha_{X}\right)$ together with a map $j_{X}: X \rightarrow U_{X}$ with the property that, for any operated semigroup $(V, \beta)$ and any map $f: X \rightarrow V$, there is a unique morphism $\bar{f}:\left(U_{X}, \alpha_{X}\right) \rightarrow(V, \beta)$ of operated semigroups such that $f=\bar{f} \circ j_{X}$. Let $\cdot$ be the binary operation on the semigroup $U_{X}$, we also use the quadruple $\left(U_{X}, \cdot, \alpha_{X}, j_{X}\right)$ to denote the free operated semigroup on $X$, except when $X=\emptyset$, when we drop $j_{X}$ and use the triple $\left(U_{X}, \cdot, \alpha_{X}\right)$.

We similarly define the concepts of free operated monoids, and free operated unitary and nonunitary k-algebras. We also similarly define the more general concept of free $\Omega$-operated monoids. See Theorem 2.1 for the precise definition.

\subsection{Outline of the paper}

In Section 2, free operated semigroups and free operated monoids are constructed in terms of Motzkin paths (Corollary 2.2). In fact, we construct free $\Omega$-operated semi- 
groups and free $\Omega$-operated monoids in terms of a natural generalization of Motzkin paths (Theorem 2.1). Through the Motzkin words, we relate the Motzkin paths with the recursively constructed bracketed words in Section 3 (Theorem 3.4). This in turn allows us to construct free operated semigroups and free operated monoids in terms of bracketed words (Corollary 3.6). In Section 4, free $\Omega$-operated semigroups are constructed in terms of vertex decorated planar rooted forests, through a natural isomorphism from the free operated semigroup of peak-free Motzkin paths to the operated semigroup of the vertex decorated planar rooted forests (Theorem 4.2).

One can regard these results on the free objects as a first step in the study of operated semigroups and operated algebras that generalizes the extensive work on semigroups [20, 22, 32, 39].

Given the recent progresses on Rota-Baxter algebra in both theoretical and applied aspects [1, 2, 10, 15-19, 24-31], it is desirable to obtain convenient constructions of free Rota-Baxter algebras. To this end, in Section 5, we put together bijections and inclusions among bracketed words, Motzkin path, vertex decorated forests and angularly decorated forests, as well as their various subsets (Theorem 5.1). These maps preserve the structure of operated semigroups. In Section 6, we use these bijections and the construction of free Rota-Baxter algebra in terms of angularly decorated rooted forests [16] to construct free Rota-Baxter algebras in terms of Motzkin paths, bracketed words and leaf decorated forests (Corollary $6.3-6.5$ ).

Notations We will use $\mathbb{N}$ to denote the set of non-negative integers. By a ring or an algebra we mean an associative unitary one unless otherwise specified. For a commutative ring $\mathbf{k}$ and a set $Y$, we use $\mathbf{k} Y$ to denote the free $\mathbf{k}$-module with basis $Y$. When $Y$ is a monoid (resp. semigroup), $\mathbf{k} Y$ carries the natural $\mathbf{k}$-algebra (resp. nonunitary k-algebra) structure. We use $\bigcup^{\bullet}$ to denote a disjoint union.

\section{Free operated semigroups and monoids in terms of Motzkin paths}

We show that free operated semigroups and monoids have a natural construction by Motzkin paths.

Recall $[12,13]$ that a Motzkin path is a lattice path in $\mathbb{N}^{2}$ from $(0,0)$ to $(n, 0)$ whose permitted steps are an up diagonal step (or up step for short) $(1,1)$, a down diagonal step (or down step) $(1,-1)$ and a horizontal step (or level step) $(1,0)$. The first few Motzkin paths are

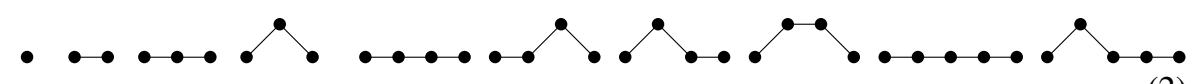

The height of a Motzkin path is simply the maximum of the height of the points on the path. Let $\mathcal{P}$ be the set of Motzkin paths. For Motzkin paths $\mathfrak{m}$ and $\mathfrak{m}^{\prime}$, define $\mathfrak{m} \circ \mathfrak{m}$, called the link product of $\mathfrak{m}$ and $\mathfrak{m}^{\prime}$, to be the Motzkin path obtained by joining the last vertex of $\mathfrak{m}$ with the first vertex of $\mathfrak{m}^{\prime}$. For example,

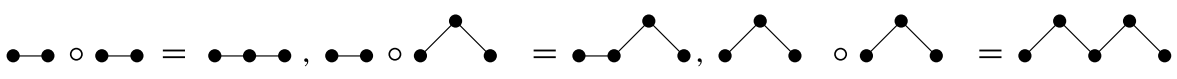


The link product is obviously associative with the trivial Motzkin path • as the identity. Let $\mathcal{I}$ be the set of indecomposable (also called prime) Motzkin paths, consisting of Motzkin paths that touch the $x$-axis only at the two end vertices. It is clear that a Motzkin path is indecomposable if and only if it is not the link product of two non-trivial Motzkin paths.

Next for a Motzkin path $\mathfrak{m}$, denote $/ \mathfrak{m} \backslash$ to be the Motzkin path obtained by raising $\mathfrak{m}$ on the left end by an up step and on the right end by a down step. For example,

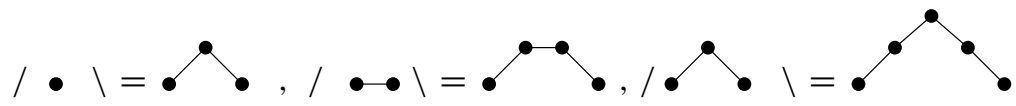

This defines an operator $/ \backslash$ on $\mathcal{P}$, called the raising operator. Thus $\mathcal{P}$, with the link product $\circ$ and the raising operator $/ \backslash$, is an operated monoid.

Let $\mathcal{D}$ denote set of Dyck paths which are defined to be paths that do not have any level steps. Then $(\mathcal{D}, \circ, / \backslash)$ is an operated submonoid of the operated monoid $(\mathcal{P}, \circ, / \backslash)$.

A Motzkin path is called peak-free if it is not - and does not have an up step followed immediately by a down step. For example,

and $\bullet \bullet \bullet$ in the list (2) are peak-free while the rest are not. Let $\mathcal{L}$ denote the set of peak-free Motzkin paths. Then $(\mathcal{L}, \circ, / \backslash)$ is an operated subsemigroup (but not submonoid) of $(\mathcal{P}, \circ, / \backslash)$.

Let $X$ be a set. An $X$-decorated (or colored) Motzkin path $[8,12]$ is a Motzkin path whose level steps are decorated (colored) by elements in $X$. Some examples are

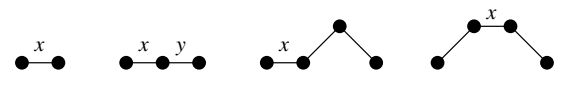

Let $\mathcal{P}(X)$ be the set of $X$-decorated Motzkin paths and let $\mathcal{L}(X)$ be the set of peakfree $X$-decorated Motzkin paths. Note that Motzkin paths with no decorations can be identified with $X$-decorated Motzkin paths where $X$ is a singleton.

We next generalize the concept of Motzkin paths to allow decorations on the up and down steps. A matching pair of steps in a Motzkin path consists of an up step and the first down step to the right of this up step with the same height. To put it another way, a matching pair of steps is an up step and a down step to its right such that the path between (and excluding) these two steps is a Motzkin path.

Let $\Omega$ be a set. By an $(X, \Omega)$-decorated or a fully decorated Motzkin path we mean a Motzkin path where each matching pair of steps is decorated by an element of $\Omega$, and where each level step is decorated by an element of $X$. For example,

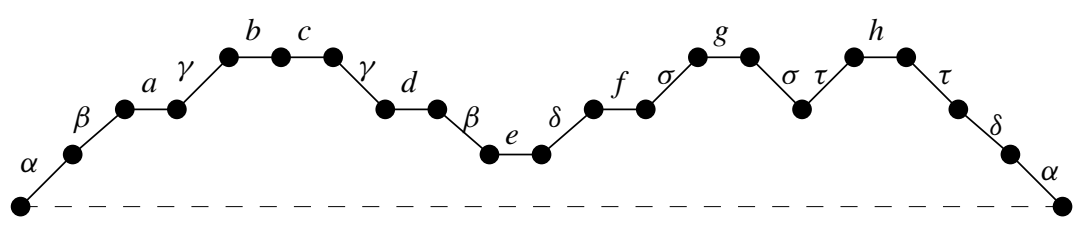

is an $(X, \Omega)$-decorated Motzkin path with $a, b, c, d, e, f, g, h \in X$ and $\alpha, \beta, \gamma, \delta, \sigma$, $\tau \in \Omega$. 
The set of $(X, \Omega)$-decorated Motzkin paths is denoted by $\mathcal{P}(X, \Omega)$. We similarly define $(X, \Omega)$-decorated peak-free Motzkin paths $\mathcal{L}(X, \Omega)$ and $\Omega$-decorated Dyck paths $\mathcal{D}(\Omega)$. The last notation makes sense since a Dyck path does not have any level steps and thus does not involve decorations by $X$.

The link product of two $(X, \Omega)$-decorated Motzkin paths is defined in the same way as for Motzkin paths. Further, for each $\omega \in \Omega$ and an $(X, \Omega)$-decorated Motzkin path $\mathfrak{m}$, we define $/ \omega \mathfrak{m} \backslash \omega$ to be the Motzkin path obtained by raising $\mathfrak{m}$ on the left end by an up step on the right end by a down step, both decorated by $\omega$. For example, we have

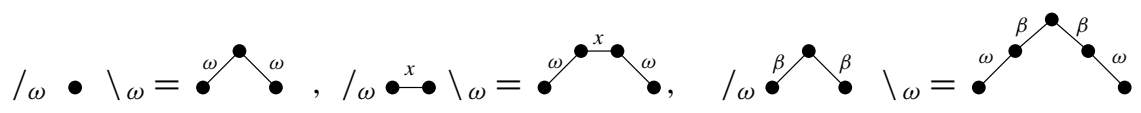

Thus for each $\omega \in \Omega$, we obtain a map $/ \omega \backslash \omega$ on each of the semigroups or monoids $\mathcal{P}(X, \Omega), \mathcal{L}(X, \Omega)$ and $\mathcal{D}(\Omega)$, making it into an $\Omega$-operated semigroup or an $\Omega$ operated monoid.

The concepts of height and indecomposability in $\mathcal{P}(X, \Omega)$ are defined in the same way as in $\mathcal{P}$. For $n \geq 0$, let $\mathcal{P}_{n}(X, \Omega)$ be the submonoid of elements of $\mathcal{P}(X, \Omega)$ of height $\leq n$. Also define $\mathcal{P}_{-1}(X, \Omega)=\emptyset$. Define $\mathcal{L}_{n}(X, \Omega)=\mathcal{P}_{n}(X, \Omega) \cap \mathcal{L}(X, \Omega)$ and $\mathcal{D}_{n}(\Omega)=\mathcal{P}_{n}(X, \Omega) \cap \mathcal{D}(\Omega)$.

Theorem 2.1 Let $\Omega$ and $X$ be non-empty sets. Let $j_{X}: X \rightarrow \mathcal{L}(X, \Omega) \subseteq \mathcal{P}(X, \Omega)$ be defined by $j_{X}(x)=\bullet^{x} \bullet, x \in X$.

(a) The quadruple $\left(\mathcal{P}(X, \Omega), \circ,\{/ \omega \backslash \omega \mid \omega \in \Omega\}, j_{X}\right)$ is the free $\Omega$-operated monoid on X. More precisely, for any $\Omega$-operated monoid $\left(H,\left\{\alpha_{\omega} \mid \omega \in \Omega\right\}\right)$ consisting of a monoid $H$ and maps $\alpha_{\omega}: H \rightarrow H$ for $\omega \in \Omega$, there is a unique morphism $\bar{f}:(\mathcal{P}(X, \Omega),\{/ \omega \backslash \omega \mid \omega \in \Omega\}) \rightarrow\left(H,\left\{\alpha_{\omega} \mid \omega \in \Omega\right\}\right)$ of operated monoids such that $f=\bar{f} \circ j_{X}$.

(b) The quadruple $\left(\mathcal{L}(X, \Omega), \circ,\{/ \omega \backslash \omega \mid \omega \in \Omega\}, j_{X}\right)$ is the free $\Omega$-operated semigroup on $X$.

(c) The triple $(\mathcal{D}(\Omega), \circ,\{/ \omega \backslash \omega \mid \omega \in \Omega\})$ is the free $\Omega$-operated monoid on the empty set.

Proof We only need to prove (a). The proof of the other parts are similar.

Let $\left(H,\left\{\alpha_{\omega} \mid \omega \in \Omega\right\}\right)$ be an $\Omega$-operated monoid with a monoid $H$ and maps $\alpha_{\omega}$ : $H \rightarrow H, \omega \in \Omega$. Let $f: X \rightarrow H$ be a set map. We will use induction on $n$ to construct a unique sequence of monoid homomorphisms

$$
\bar{f}_{n}: \mathcal{P}_{n}(X, \Omega) \rightarrow H, n \geq 0,
$$

with the following properties.

(a) $\left.\bar{f}_{n}\right|_{\mathcal{P}_{n-1}(X, \Omega)}=\bar{f}_{n-1}$.

(b) $\bar{f}_{n} \circ(/ \omega \backslash \omega)=\alpha_{\omega} \circ \bar{f}_{n-1}$ on $\mathcal{P}_{n-1}(X, \Omega)$ for each $\omega \in \Omega$.

When $n=0, \mathcal{P}_{0}(X, \Omega)$ is the monoid of paths from $(0,0)$ to $(m, 0), m \geq 0$, consisting of only level steps which are decorated by elements of $X$. Thus $\overline{\mathcal{P}}_{0}(X, \Omega)$ 
is the free monoid generated by $\left\{\bullet^{x} \bullet \mid x \in X\right\}$. Then the map $f: X \rightarrow H$ extends uniquely to a monoid homomorphism $\bar{f}_{0}: \mathcal{P}_{0}(X, \Omega) \rightarrow H$ such that $\bar{f}_{0} \circ j_{X}=f . \bar{f}_{0}$ trivially satisfies properties (a) and (b) since $\mathcal{P}_{-1}(X, \Omega)=\emptyset$ by convention.

For given $k \geq 0$, assume that there is a unique map $\bar{f}_{k}: \mathcal{P}_{k}(X, \Omega) \rightarrow H$ satisfying the properties (a) and (b). Note that $\mathcal{P}_{k+1}(X, \Omega)$ is the free monoid generated by $\mathcal{I}_{k+1}(X, \Omega)$, the set of indecomposable Motzkin paths of height $\leq k+1$, and note that an indecomposable Motzkin path of height $k+1$ is of the form $/ \omega \overline{\mathfrak{m}} \backslash \omega$ for an $\omega \in \Omega$ and an $\overline{\mathfrak{m}} \in \mathcal{P}_{k}(X, \Omega)$ of height $k$. This is because an $\mathfrak{m} \in \mathcal{I}_{k+1}(X, \Omega)$ touches the $x$-axis only at the beginning and the end of the path. So the first step must be a rise step and the last step must be a fall step, decorated by the same $\omega \in \Omega$. Further, if the first step and the last step are removed, we still have a Motzkin path $\overline{\mathfrak{m}}$ of height $k$ and $\mathfrak{m}=/ \omega \overline{\mathfrak{m}} \backslash \omega$.

Thus we have the disjoint union

$$
\mathcal{I}_{k+1}(X, \Omega)=\mathcal{I}_{k}(X, \Omega) \dot{\bigcup}\left(\bigcup_{\omega \in \Omega / \omega}\left(\mathcal{L}_{k}(X, \Omega) \backslash \mathcal{L}_{k-1}(X, \Omega)\right) \backslash \omega\right) .
$$

Define $f_{k+1}: \mathcal{I}_{k+1}(X, \Omega) \rightarrow H$ by requiring

$$
f_{k+1}(\mathfrak{m})= \begin{cases}\bar{f}_{k}(\mathfrak{m}), & \mathfrak{m} \in \mathcal{I}_{k}(X, \Omega), \\ \alpha_{\omega}\left(\overline{f_{k}}(\overline{\mathfrak{m}})\right), & \mathfrak{m}=/ \omega \overline{\mathfrak{m}} \backslash_{\omega} \in / \omega\end{cases}
$$

Then extend $f_{k+1}$ to the free monoid $\mathcal{P}_{k+1}(X, \Omega)$ on $\mathcal{I}_{k+1}(X, \Omega)$ by multiplicity and obtain

$$
\bar{f}_{k+1}: \mathcal{P}_{k+1}(X, \Omega) \rightarrow H .
$$

By the construction of $f_{k+1}, \bar{f}_{k+1}$ satisfies properties (a) and (b), and it is the unique such monoid homomorphism.

By Property (a), the sequence $\left\{\bar{f}_{n}, n \geq 0\right\}$ forms a direct system of monoid homomorphisms and thus gives the direct limit

$$
\bar{f}=\lim _{\underline{f}} \bar{f}_{n} \mathcal{P}(X, \Omega) \rightarrow H
$$

which is naturally a monoid homomorphism. By Property (b), we have $\bar{f} \circ(/ \omega \backslash \omega)=$ $\alpha_{\omega} \circ \bar{f}, \forall \omega \in \Omega$. Thus $\bar{f}$ is a homomorphism of $\Omega$-operated monoids such that $\bar{f} \circ j_{X}=f$.

Furthermore, if $\bar{f}^{\prime}: \mathcal{P}(X, \Omega) \rightarrow H$ is another homomorphism of $\Omega$-operated monoids such that $\bar{f}^{\prime} \circ j_{X}=f$. Let $\bar{f}_{n}^{\prime}=\left.\bar{f}^{\prime}\right|_{\mathcal{P}_{n}(X, \Omega)}$. Then we have

$$
\bar{f}^{\prime} \circ j_{X}=f=\bar{f} \circ j_{X}
$$

and hence $\bar{f}_{0}^{\prime}=\bar{f}_{0}$ since $\mathcal{P}_{0}(X, \Omega)$ is the free monoid generated by $j_{X}(X)$. Further, $\left\{\bar{f}_{n}^{\prime}, n \geq 0\right\}$ also satisfies Property (a) by its construction, and satisfies Property (b) since $\bar{f}^{\prime}$ is a homomorphism of $\Omega$-operated monoids. But by our inductive construction of $\left\{\bar{f}_{n}, n \geq 0\right\}$, such $\bar{f}_{n}$ are unique. Thus we have $\bar{f}_{n}^{\prime}=\bar{f}_{n}, n \geq 0$, and therefore $\bar{f}=\bar{f}^{\prime}$. This proves the uniqueness of $\bar{f}$.

By taking $\Omega$ to be a singleton in Theorem 2.1, we have: 


\section{Corollary 2.2}

(a) Let $X$ be a non-empty set. The quadruple $\left(\mathcal{P}(X), \circ, / \backslash, j_{X}\right)$ is the free operated monoid on $X$. In particular, $(\mathcal{P}, \circ, / \backslash)$ is the free operated monoid on one generator.

(b) Let $X$ be a non-empty set. The quadruple $\left(\mathcal{L}(X), \circ, / \backslash, j_{X}\right)$ is the free operated semigroup on $X$. In particular, $(\mathcal{L}, \circ, / \backslash)$ is the free operated semigroup on one generator.

(c) The triple $(\mathcal{D}, \circ, / \backslash)$ is the free operated monoid on the empty set.

Recall that for a semigroup (resp. monoid) $Y$, we use $\mathbf{k} Y$ to denote the corresponding nonunitary (resp. unitary) k-algebra. We then have

Corollary 2.3 Let $\Omega$ and $X$ be non-empty sets. Let $j_{X}$ be as defined in Theorem 2.1.

(a) The quadruple $\left(\mathbf{k} \mathcal{P}(X, \Omega), \circ,\{/ \omega \backslash \omega \mid \omega \in \Omega\}, j_{X}\right)$ is the free $\Omega$-operated $\mathbf{k}$ algebra on $X$.

(b) The quadruple $\left(\mathbf{k} \mathcal{L}(X, \Omega), \circ,\{/ \omega \backslash \omega \mid \omega \in \Omega\}, j_{X}\right)$ is the free $\Omega$-operated nonunitary k-algebra on $X$.

(c) The quadruple $\left(\mathcal{D}(\Omega), \circ,\{/ \omega \backslash \omega \mid \omega \in \Omega\}, j_{X}\right)$ is the free $\Omega$-operated $\mathbf{k}$-algebra on the empty set.

Proof (a). The forgetful functor from the category $\Omega$-OAlg of $\Omega$-operated algebras to the category Set of sets is the composition of the forgetful functor from $\Omega$-OAlg to the category $\Omega$-OMon of operated monoids and the forgetful functor from $\Omega$-OMon to Set. As is well-known (for example from Theorem 1 in page 101 of [35]), the adjoint functor of a composed functor is the composition of the adjoint functors. This proves (a).

The proofs of the others parts are the same.

\section{Free operated semigroups and monoids in terms of bracketed words}

\subsection{Motzkin words}

We recall the following definition $[3,21,38]$.

Definition 3.1 A word from the alphabet set $X \cup\{/, \backslash\}$ (often denoted by $X \cup$ $\{U, D\})$ is called an $X$-decorated Motzkin word if it has the properties that

(a) the number of / in the word equals the number of $\backslash$ in the word;

(b) counting from the left, the number of occurrence of / is always greater or equal to the number of occurrence of $\backslash$.

Thus an $X$-decorated Motzkin word is an element in the free monoid $M(X \cup\{/, \backslash\})$ on the set $X \cup\{/, \backslash\}$ with above two properties. $X$-decorated Motzkin words are 
used to code Motzkin paths so that every up (resp. down) step in a Motzkin path corresponds to the symbol / (resp. $\backslash$ ) and every level step decorated by $x \in X$ corresponds to $x$. Under this coding, the set of Dyck paths corresponds to the set of legal bracketings $[5,6]$, consisting of words from the alphabet set $\{/, \backslash\}$ with the above two properties.

We now generalize the concept of decorated Motzkin words. Consider the free monoid

$$
M_{X, \Omega}=M(X \cup\{/ \omega \mid \omega \in \Omega\} \cup\{\backslash \omega \mid \omega \in \Omega\})
$$

on the set $X \cup\{/ \omega \mid \omega \in \Omega\} \cup\{\backslash \omega \mid \omega \in \Omega\}$. For a given $\omega \in \Omega$, define

$$
P_{\omega}: M_{X, \Omega} \rightarrow M_{X, \Omega}, \quad P_{\omega}(\mathfrak{m})=/{ }_{\omega} \mathfrak{m} \backslash \omega, \mathfrak{m} \in M_{X, \Omega} .
$$

Then $M_{X, \Omega}$ is an $\Omega$-operated monoid. Define $\mathcal{W}(X, \Omega)$ to be the $\Omega$-operated submonoid of $M_{X, \Omega}$ generated by $X$. Elements of $\mathcal{W}(X, \Omega)$ are called $(X, \Omega)$-decorated Motzkin words.

We next show that, as in the case of Motzkin words, $(X, \Omega)$-decorated Motzkin words code $(X, \Omega)$-decorated Motzkin paths.

Proposition 3.2 The $\Omega$-operated monoids $\mathcal{P}(X, \Omega)$ and $\mathcal{W}(X, \Omega)$ are isomorphic. Consequently, $\mathcal{W}(X, \Omega)$ is the free $\Omega$-operated monoid on $X$.

Proof By the freeness of $\mathcal{P}(X, \Omega)$, there is a unique $\Omega$-operated monoid homomorphism

$$
\phi_{\mathcal{P}, \mathcal{W}}: \mathcal{P}(X, \Omega) \rightarrow \mathcal{W}(X, \Omega)
$$

such that $\phi_{\mathcal{P}, \mathcal{W}}\left(\bullet^{x} \bullet\right)=x, x \in X$. This homomorphism is surjective since $\mathcal{W}(X, \Omega)$ is generated by $X$ as an $\Omega$-operated monoid.

To see the injectivity of $\phi_{\mathcal{P}, \mathcal{W}}$, we only need to note that $\phi_{\mathcal{P}, \mathcal{W}}$ sends

$$
\left\{\begin{array}{l}
\text { an up step in a Motzkin path decorated by } \omega \in \Omega \text { to } / \omega, \\
\text { a down step in a Motzkin path decorated by } \omega \in \Omega \text { to } \backslash \omega, \\
\text { a level step in a Motzkin path decorated by } x \in X \text { to } x
\end{array}\right.
$$

Thus distinct Motzkin paths correspond to distinct Motzkin words.

Remark 3.3 Through the bijection $\phi_{\mathcal{P}, \mathcal{W}}$, we can use the definition of a $(X, \Omega)$ Motzkin path to characterize an $(X, \Omega)$-Motzkin word to be a word $\mathfrak{w} \in M_{X, \Omega}$ such that

(a) ignoring the $\Omega$-decoration of $\mathfrak{w}$, we have an $X$-decorated Motzkin word;

(b) for any letter / in $\mathfrak{w}$ decorated an $\omega \in \Omega$, its conjugate $\backslash$ is also decorated by the same $\omega$.

Here for each / in $\mathfrak{w}$, the conjugate of / is the $\backslash$ in $\mathfrak{w}$ to the right of this / such that the subword of $\mathfrak{w}$ between (and excluding) these / and $\backslash$ is a $X$-decorated Motzkin word. The existence and uniqueness of the conjugate follow from the matching down step of an up step in the matching pair of Motzkin paths. 


\subsection{Bracketed words}

We use the following recursion to give an external construction of $(X, \Omega)$-decorated Motzkin words and hence of the free operated semigroup and free operated monoid over $X$.

For any set $Y$, let $S(Y)$ denote the free semigroup generated by $Y$, let $M(Y)$ denote the free monoid generated by $Y$. For a fixed $\omega \in \Omega$, let $\left\lfloor{ }_{\omega} Y\right\rfloor_{\omega}$ denote the set $\left\{\lfloor\omega y\rfloor_{\omega} \mid y \in Y\right\}$ which is in bijection with $Y$, but disjoint from $Y$. Also assume the sets $\lfloor\omega Y\rfloor_{\omega}$ to be disjoint with each other as $\omega \in \Omega$ varies.

We now inductively define a direct system $\left\{\mathcal{S}_{n}=\mathcal{S}_{n}(X, \Omega), i_{n, n+1}: \mathcal{S}_{n} \rightarrow\right.$ $\left.\mathcal{S}_{n+1}\right\}_{n \in \mathbb{N}}$ of free semigroups and a direct system $\left\{\mathcal{M}_{n}=\mathcal{M}_{n}(X, \Omega), \tilde{i}_{n, n+1}: \mathcal{M}_{n} \rightarrow\right.$ $\left.\mathcal{M}_{n+1}\right\}_{n \in \mathbb{N}}$ of free monoids, both with inclusions as the transition maps, and such that, for each $\omega \in \Omega$,

$$
\left\lfloor{ }_{\omega} \mathcal{S}_{n}\right\rfloor_{\omega} \subseteq \mathcal{S}_{n+1}, \quad\left\lfloor\omega \mathcal{M}_{n}\right\rfloor_{\omega} \subseteq \mathcal{M}_{n+1}, n \in \mathbb{N} .
$$

We do this by first defining $\mathcal{S}_{0}:=S(X)$ and $\mathcal{M}_{0}:=M(X)=S(X) \cup\{\mathbf{1}\}$, and then defining

$$
\begin{aligned}
& \mathcal{S}_{1}:=S\left(X \cup\left(\cup_{\omega \in \Omega}\left\lfloor_{\omega} \mathcal{S}_{0}\right\rfloor_{\omega}\right)\right)=S\left(X \cup\left(\cup_{\omega \in \Omega}\left\lfloor_{\omega} S(X)\right\rfloor_{\omega}\right)\right), \\
& \mathcal{M}_{1}:=M\left(X \cup\left(\cup_{\omega \in \Omega}\left\lfloor\omega \mathcal{M}_{0}\right\rfloor_{\omega}\right)\right)
\end{aligned}
$$

with $i_{0,1}$ and $\tilde{i}_{0,1}$ being the inclusions

$$
\begin{aligned}
& i_{0,1}: \mathcal{S}_{0}=S(X) \hookrightarrow \mathcal{S}_{1}=S\left(X \cup\left(\cup_{\omega \in \Omega}\left\lfloor_{\omega} \mathcal{S}_{0}\right\rfloor_{\omega}\right)\right), \\
& \tilde{i}_{0,1}: \mathcal{M}_{0}=M(X) \hookrightarrow \mathcal{M}_{1}=M\left(X \cup\left(\cup_{\omega \in \Omega}\left\lfloor\omega \mathcal{M}_{0}\right\rfloor_{\omega}\right)\right) .
\end{aligned}
$$

Clearly, $\left\lfloor_{\omega} \mathcal{S}_{0}\right\rfloor_{\omega} \subseteq \mathcal{S}_{1}$ and $\left\lfloor_{\omega} \mathcal{M}_{0}\right\rfloor_{\omega} \subseteq \mathcal{M}_{1}$ for each $\omega \in \Omega$.

Inductively assume that $\mathcal{S}_{n-1}$ and $\mathcal{M}_{n-1}$ have been defined for $n \geq 2$, with the inclusions

$$
i_{n-2, n-1}: \mathcal{S}_{n-2} \hookrightarrow \mathcal{S}_{n-1} \text { and } \tilde{i}_{n-2, n-1}: \mathcal{M}_{n-2} \rightarrow \mathcal{M}_{n-1} .
$$

We then define

$$
\mathcal{S}_{n}:=S\left(X \cup\left(\cup_{\omega \in \Omega}\left\lfloor{ }_{\omega} \mathcal{S}_{n-1}\right\rfloor_{\omega}\right)\right) \text { and } \mathcal{M}_{n}:=M\left(X \cup\left(\cup_{\omega \in \Omega}\left\lfloor{ }_{\omega} \mathcal{M}_{n-1}\right\rfloor_{\omega}\right)\right) .
$$

The inclusions in Eq. (9) give the inclusions

$$
\left\lfloor\omega \mathcal{S}_{n-2}\right\rfloor_{\omega} \hookrightarrow\left\lfloor{ }_{\omega} \mathcal{S}_{n-1}\right\rfloor_{\omega} \text { and }\left\lfloor{ }_{\omega} \mathcal{M}_{n-2}\right\rfloor_{\omega} \hookrightarrow\left\lfloor{ }_{\omega} \mathcal{M}_{n-1}\right\rfloor_{\omega},
$$

yielding inclusions of free semigroups and free monoids

$$
\begin{aligned}
i_{n-1, n}: \mathcal{S}_{n-1} & =S\left(X \cup\left(\cup_{\omega \in \Omega}\left\lfloor_{\omega} \mathcal{S}_{n-2}\right\rfloor_{\omega}\right)\right) \hookrightarrow S\left(X \cup\left(\cup_{\omega \in \Omega}\left\lfloor_{\omega} \mathcal{S}_{n-1}\right\rfloor_{\omega}\right)\right)=\mathcal{S}_{n}, \\
\tilde{i}_{n-1, n}: \mathcal{M}_{n-1} & =M\left(X \cup \left(\cup_{\omega \in \Omega}\left\lfloor{ }_{\omega} \mathcal{M}_{n-2}\right\rfloor \omega\right.\right. \\
& =\mathcal{M}_{n} .
\end{aligned}
$$


By Eq. (10), $\left.\rfloor_{\omega} \mathcal{S}_{n-1}\right\rfloor_{\omega} \subseteq \mathcal{S}_{n}$ and $\left\lfloor{ }_{\omega} \mathcal{M}_{n-1}\right\rfloor_{\omega} \subseteq \mathcal{M}_{n}$. This completes the inductive construction of the direct systems. Define the direct limit of semigroups

$$
\mathcal{S}(X, \Omega)=\underline{\lim } \mathcal{S}_{n}=\bigcup_{n \geq 0} \mathcal{S}_{n}
$$

whose elements are called nonunitary bracketed words and the direct limit of monoids

$$
\mathcal{M}(X, \Omega)=\underset{\lim }{\longrightarrow} \mathcal{M}_{n}=\bigcup_{n \geq 0} \mathcal{M}_{n}
$$

whose elements are called unitary bracketed words. Then by Eq. (8), $\left\lfloor{ }_{\omega} \mathcal{S}(X, \Omega)\right\rfloor_{\omega} \subseteq \mathcal{S}(X, \Omega)$ and $\left\lfloor_{\omega} \mathcal{M}(X, \Omega)\right\rfloor_{\omega} \subseteq \mathcal{M}(X, \Omega)$ for $\omega \in \Omega$. Thus $\mathcal{S}(X, \Omega)$ (resp. $\mathcal{M}(X, \Omega)$ ) carries an $\Omega$-operated semigroup (resp. monoid) structure.

\section{Theorem 3.4}

(a) The $\Omega$-operated monoids $\mathcal{M}(X, \Omega)$ and $\mathcal{W}(X, \Omega)$ are naturally isomorphic.

(b) The $\Omega$-operated monoids $\mathcal{M}(X, \Omega)$ and $\mathcal{P}(X, \Omega)$ are naturally isomorphic.

(c) The $\Omega$-mapped semigroups $\mathcal{S}(X, \Omega)$ and $\mathcal{L}(X, \Omega)$ are naturally isomorphic.

Proof (a) By Theorem 2.1 and Proposition 3.2, $(\mathcal{W}(X), \circ, / \backslash)$ is a free $\Omega$-operated monoid on $X$. Thus there is a unique homomorphism of $\Omega$-operated monoids

$$
\phi_{\mathcal{W}, \mathcal{M}}: \mathcal{W}(X, \Omega) \rightarrow \mathcal{M}(X, \Omega)
$$

such that $\phi_{\mathcal{W}, \mathcal{M}}(x)=x$.

Let $\mathcal{M}^{\prime}$ be the $\Omega$-operated submonoid of $\mathcal{M}(X, \Omega)$ generated by $X$. Then an inductive argument shows that $\mathcal{M}_{n} \subseteq \mathcal{M}^{\prime}$ for all $n \geq 0$. Thus the $\Omega$-operated monoid $\mathcal{M}(X, \Omega)$ is generated by $X$ and therefore $\phi_{\mathcal{W}, \mathcal{M}}$ is surjective.

To prove that $\phi_{\mathcal{W}, \mathcal{M}}$ is injective, we only need to define a homomorphism of $\Omega$-operated monoids

$$
\phi_{\mathcal{M}, \mathcal{W}}: \mathcal{M}(X, \Omega) \rightarrow \mathcal{W}(X, \Omega)
$$

such that $\phi_{\mathcal{M}, \mathcal{W}} \circ \phi_{\mathcal{W}, \mathcal{M}}=\mathrm{i} d_{\mathcal{W}(X, \Omega)}$. For this, we inductively define monoid homomorphisms

$$
\phi_{\mathcal{M}, \mathcal{W}, n}: \mathcal{M}_{n} \rightarrow \mathcal{W}(X, \Omega), \quad n \geq 0
$$

by taking

(a) $\phi_{\mathcal{M}, \mathcal{W}, 0}$ to be the unique monoid homomorphism from the free monoid $\mathcal{M}_{0}$ on $X$ to $\mathcal{W}(X, \Omega)$ sending $x$ to $x, x \in X$, and

(b) $\phi_{\mathcal{M}, \mathcal{W}, k+1}$ to be such that $\phi_{\mathcal{M}, \mathcal{W}, k+1}\left(\left\lfloor_{\omega} \mathfrak{m}\right\rfloor_{\omega}\right)=/{ }_{\omega} \phi_{\mathcal{M}, \mathcal{W}, n}(\mathfrak{m}) \backslash \omega, \mathfrak{m} \in \mathcal{M}_{n}$. Here $\phi_{\mathcal{M}, \mathcal{W}, n}(\mathfrak{m})$ is defined by the induction hypothesis.

Taking the direct limit, we obtain a monoid homomorphism.

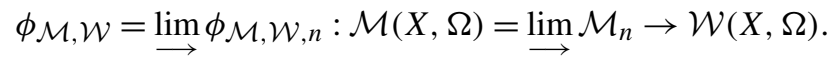


By item (b) above, for each $\omega \in \Omega$, the bracket operator $\left.\iota_{\omega}\right\rfloor_{\omega}$ on $\mathcal{M}(X, \Omega)$ is com-

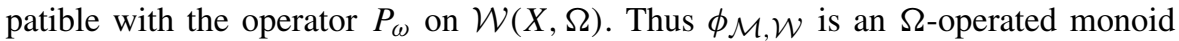
homomorphism. Further, since $\phi_{\mathcal{M}, \mathcal{W}} \circ \phi_{\mathcal{W}, \mathcal{M}}$ is the identity on $X$, by the universal property of $\mathcal{W}(X, \Omega)$, we must have $\phi_{\mathcal{M}, \mathcal{W}} \circ \phi_{\mathcal{W}, \mathcal{M}}=\mathrm{i} d_{\mathcal{W}(X, \Omega)}$.

(b) The isomorphism is

$$
\phi_{\mathcal{M}, \mathcal{P}}=\phi_{\mathcal{P}, \mathcal{W}}^{-1} \circ \phi_{\mathcal{M}, \mathcal{W}}
$$

for $\phi_{\mathcal{P}, \mathcal{W}}$ in Eq. (6) and $\phi_{\mathcal{M}, \mathcal{W}}$ in Eq. (14).

(c) Since $\mathcal{S}(X, \Omega)$ (resp. $\mathcal{L}(X, \Omega)$ ) is the $\Omega$-operated subsemigroup of $\mathcal{M}(X, \Omega)$ (resp. $\mathcal{P}(X, \Omega)$ ) generated by $X$ (resp. $\left\{\bullet^{x} \bullet \mid x \in X\right\}$ ), the $\Omega$-operated monoid isomorphism $\phi_{\mathcal{M}, \mathcal{P}}$ in Eq. (16) restricts to an isomorphism $\phi_{\mathcal{S}, \mathcal{L}}: \mathcal{S}(X, \Omega) \rightarrow \mathcal{L}(X, \Omega)$ of $\Omega$-operated semigroups.

Remark 3.5 In the proof of Theorem 3.4, the isomorphism $\phi_{\mathcal{M}, \mathcal{W}}: \mathcal{M}(X, \Omega) \rightarrow$ $\mathcal{W}(X, \Omega)$ is almost like the identity map. For example,

$$
\phi_{\mathcal{M}, \mathcal{W}}\left(x\left\lfloor_{\omega} y\right\rfloor_{\omega}\right)=\phi_{\mathcal{M}, \mathcal{W}}(x) \phi_{\mathcal{M}, \mathcal{W}}\left(\left\lfloor_{\omega} y\right\rfloor_{\omega}\right)=x / \omega y \backslash \omega
$$

The difference is that in $x\left\lfloor_{\omega} y\right\rfloor_{\omega},\lfloor\omega y\rfloor_{\omega}$ is a new symbol, while in $x / \omega y \backslash \omega$, / $\omega y \backslash \omega$ is a word consisting of the three symbols $/ \omega, y$ and $\backslash \omega$. Thus we can identify $\mathcal{M}(X, \Omega)$ with $\mathcal{W}(X, \Omega)$, allowing us to use $\mathcal{M}(X, \Omega)$ to give a recursive description of $\mathcal{W}(X, \Omega)$ and use $\mathcal{W}(X, \Omega)$ to give an explicit description of $\mathcal{M}(X, \Omega)$.

By Theorem 2.1 and Theorem 3.4, we have

Corollary 3.6 Let $j_{X}$ denote the natural embeddings $X \rightarrow \mathcal{M}(X, \Omega)$ or $X \rightarrow$ $\mathcal{S}(X, \Omega)$.

(a) With the word concatenation product, the triple $\left(\mathcal{M}(X, \Omega),\llcorner\rfloor, j_{X}\right)$ is the free $\Omega$-operated monoid on $X$.

(b) With the word concatenation product, the triple $\left(\mathcal{S}(X, \Omega),\lfloor\rfloor, j_{X}\right)$ is the free $\Omega$ operated semigroup on $X$.

We will use $j_{X}$ here and later to denote the natural embeddings of $X$ to various operated monoids and operated semigroups. The meaning will be clear from the context. By the same proof as for Corollary 2.3, we further have:

Corollary 3.7 Let $j_{X}$ denote the natural embeddings from $X$ to $\mathbf{k} \mathcal{M}(X)$ or $\mathbf{k} \mathcal{S}(X)$.

(a) The triple $\left(\mathbf{k} \mathcal{M}(X),\lfloor\rfloor, j_{X}\right)$ is the free operated (unitary) $\mathbf{k}$-algebra on $X$.

(b) The triple $\left(\mathbf{k} \mathcal{S}(X),\lfloor\rfloor, j_{X}\right)$ is the free operated nonunitary $\mathbf{k}$-algebra on $X$. 


\section{Free operated semigroups in terms of planar rooted trees}

We follow the notations and terminologies in [11, 43]. A free tree is an undirected graph that is connected and contains no cycles. A rooted tree is a free tree in which a particular vertex has been distinguished as the root. A planar rooted tree (or ordered tree) is a rooted tree with a fixed embedding into the plane. The depth $\mathrm{d}(T)$ of a rooted tree $T$ is the length of the longest path from its root to its leaves.

Let $\mathcal{T}$ be the set of planar rooted trees. A planar rooted forest is a noncommutative concatenation of planar rooted trees, denoted by $T_{1} \sqcup \cdots \sqcup T_{b}$ or simply $T_{1} \cdots T_{b}$, with $T_{1}, \cdots, T_{b} \in \mathcal{T}$. The depth $\mathrm{d}(F)$ of $F$ is the maximum of the depths of the trees $T_{i}, 1 \leq i \leq b$. Let $\mathcal{F}$ be the set of planar rooted forests. Then $\mathcal{F}$ is the free semigroup generated by $\mathcal{T}$ with the tree concatenation product.

Remark 4.1 For the rest of this paper, a tree or forest means a planar rooted tree or a planar rooted forest unless otherwise specified.

Let $\left\lfloor T_{1} \cdots T_{b}\right\rfloor$ denote the usual grafting of the trees $T_{1}, \cdots, T_{b}$ by adding a new root together with an edge from the new root to the root of each of the trees $T_{1}, \cdots, T_{b}$.

For two non-empty sets $X$ and $\Omega$, let $\mathcal{F}(X, \Omega)$ be the set of planar rooted forests whose leaf vertices are decorated by elements of $X$ and non-leaf vertices are decorated by elements of $\Omega$. The only vertex of the tree $\bullet$ is taken to be a leaf vertex. For example,

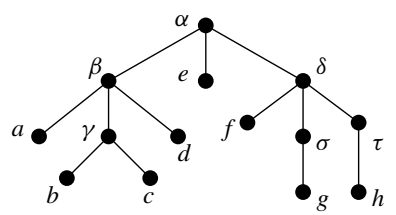

with $a, b, c, d, e, f, g, h \in X$ and $\alpha, \beta, \gamma, \delta, \sigma, \tau \in \Omega$. As special cases, we have $\mathcal{F}(X, X)$ of planar rooted forests whose vertices are decorated by elements of $X$. When $X=\Omega=\{x\}, \mathcal{F}(X, X)$ is identified with the planar rooted forests without decorations.

As in the above case of planar rooted forests without decorations, $\mathcal{F}(X, \Omega)$, with the concatenation product, is a semigroup. Further, for $\omega \in \Omega$ and $F=T_{1} \cdots T_{b} \in$ $\mathcal{F}(X, \Omega)$, let $\left\lfloor_{\omega} F\right\rfloor_{\omega}$ be the grafting of $T_{1}, \cdots, T_{b}$ with the new root decorated by $\omega$. Then with the grafting operators $\left\lfloor_{\omega}\right\rfloor_{\omega}, \omega \in \Omega, \mathcal{F}(X, \Omega)$ is an $\Omega$-operated semigroup.

We now describe the recursive structure on $\mathcal{F}(X, \Omega)$ in algebraic terms. For any subset $Y$ of $\mathcal{F}(X, \Omega)$, let $\langle Y\rangle$ be the sub-semigroup of $\mathcal{F}(X, \Omega)$ generated by $Y$. Let $\mathcal{F}_{0}(X, \Omega)=\left\langle\left\{\bullet_{x} \mid x \in X\right\}\right\rangle$, consisting of forests composed of trees $\bullet_{x}, x \in X$. These forests are also the ones decorated by $X$ of depth zero. Then recursively define

$$
\mathcal{F}_{n}(X, \Omega)=\left\langle X \cup\left(\cup_{\omega \in \Omega}\left\lfloor_{\omega} \mathcal{F}_{n-1}(X, \Omega)\right\rfloor_{\omega}\right)\right\rangle .
$$

It is clear that $\mathcal{F}_{n}(X, \Omega)$ is the set of $(X, \Omega)$-decorated forests with depth less or equal to $n$ and

$$
\mathcal{F}(X, \Omega)=\cup_{n \geq 0} \mathcal{F}_{n}(X, \Omega)=\lim _{\longrightarrow} \mathcal{F}_{n}(X, \Omega) .
$$


Theorem 4.2 Let $X$ and $\Omega$ be non-empty sets.

(a) $\mathcal{F}(X, \Omega)$ is the free $\Omega$-operated semigroup on $X$.

(b) $\boldsymbol{k} \mathcal{F}(X, \Omega)$ is the free $\Omega$-operated non-unitary algebra on $X$.

Proof (a) It can be proved directly following the proof of Theorem 2.1.(b), using the recursive structure on $\mathcal{F}(X, \Omega)$ in Eq. (18). Just replace $\mathcal{L}(X, \Omega)$ and $\mathcal{L}_{n}(X, \Omega), n \geq$ 0 by $\mathcal{F}(X, \Omega)$ and $\mathcal{F}_{n}(M, \Omega)$. We leave the details to the interested reader and turn to an indirect proof by showing that the $\Omega$-operated semigroup $\mathcal{F}(X, \Omega)$ is isomorphic to the $\Omega$-operated semigroup $\mathcal{L}(X, \Omega)$. Hence $\mathcal{F}(X, \Omega)$ is free by Theorem 2.1.(b).

We obtain such an isomorphism by starting with the natural set map

$$
f: X \rightarrow \mathcal{F}(X, \Omega), x \mapsto \bullet x, x \in X
$$

Then by Theorem 2.1.(b), there is a unique homomorphism

$$
\phi_{\mathcal{L}, \mathcal{F}}: \mathcal{L}(X, \Omega) \rightarrow \mathcal{F}(X, \Omega)
$$

of $\Omega$-operated semigroups such that $\phi_{\mathcal{L}, \mathcal{F}}\left(\bullet^{x} \bullet\right)=\bullet{ }_{x}, x \in X$. We only need to show that $\phi_{\mathcal{L}, \mathcal{F}}$ is bijective.

By an inductive argument, the $\Omega$-operated subsemigroup of $\mathcal{F}(X, \Omega)$ generated by $X$ contains $\mathcal{F}_{n}(X, \Omega)$ for all $n \geq 0$. Thus by Eq. $(18), \mathcal{F}(X, \Omega)$ is generated by $X$ as an $\Omega$-operated semigroup. Thus $\phi_{\mathcal{L}, \mathcal{F}}$ is surjective. To prove that $\phi_{\mathcal{L}, \mathcal{F}}$ is injective, we construct a homomorphism of $\Omega$-operated semigroups

$$
\phi_{\mathcal{F}, \mathcal{L}}: \mathcal{F}(X, \Omega) \rightarrow \mathcal{L}(X, \Omega)
$$

such that $\phi_{\mathcal{F}, \mathcal{L}} \circ \phi_{\mathcal{L}, \mathcal{F}}=\mathrm{i} d_{\mathcal{L}(X, \Omega)}$. This follows by multiplicity from a map

$$
\phi_{\mathcal{F}, \mathcal{L}}: \mathcal{T}(X, \Omega) \rightarrow \mathcal{L}(X, \Omega) \cap \mathcal{I}(X, \Omega)
$$

This explicitly defined combinatorial bijection might be of interest on its own right. Trees and Motzkin paths have been related in previous works such as [12].

To define $\phi_{\mathcal{F}, \mathcal{L}}$ in Eq. (22), we first combine the well-known processes of preorder and postorder of traversing a planar rooted tree to define the process of biorder. The vertex biorder list of a tree $T \in \mathcal{T}(X, \Omega)$ is defined as follows.

(a) If $T$ has only one vertex, then that vertex is the vertex biorder list of $T$;

(b) If $T$ has more than one vertices, then the root vertex of $T$ has branches $T_{1}, \cdots, T_{k}, k \geq 1$, listed from the left to the right. Then the vertex biorder list of $T$ is the root of $T$, followed by the vertex biorder list of $T_{1}, \cdots$, followed by the vertex biorder list of $T_{k}$, followed by the root of $T$.

We use the adjective vertex with biorder to distinguish it from the edge biorder list to be introduced in Remark 5.2. For example, the vertex biorder list of the tree in Eq. (17) is

$$
\alpha \beta a \gamma b c \gamma d \beta e \delta f \sigma g \sigma \tau h \tau \delta \alpha
$$


It is clear that a vertex appears exactly once in the list if it is a leaf and exactly twice if it is not a leaf. The only vertex of $\bullet$ is taken to be a leaf. Thus we can record $/ \omega$ (instead of $\omega$ ) if a non-leaf vertex decorated by $\omega$ is listed for the first time and $\backslash \omega$ (instead of $\omega$ ) if this vertex is listed for the second time. This gives a word in $M_{X, \Omega}$ defined in Eq. (5) and satisfies the required properties of an $(X, \Omega)$-Motzkin word described in Remark 3.3 and therefore gives an $(X, \Omega)$-Motzkin word. For example, the Motzkin word from Eq. (23) is

$$
/_{\alpha} /{ }_{\beta} a /{ }_{\gamma} b c \backslash_{\gamma} d \backslash_{\beta} e / \delta f / \sigma g \backslash_{\sigma} /{ }_{\tau} h{ }_{\tau} \backslash_{\delta} \backslash_{\alpha}
$$

Then through the bijection $\phi_{\mathcal{P}, \mathcal{W}}$ in Eq. (7), such an $(X, \Omega)$-decorated Motzkin word gives an $(X, \Omega)$-decorated Motzkin path. As an example, the Motzkin word in Eq. (24) above corresponds to the Motzkin path

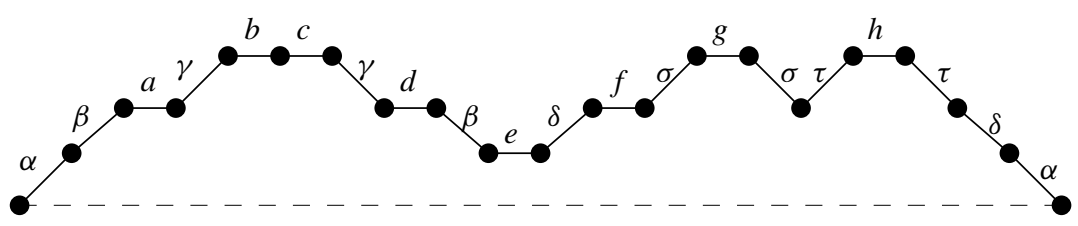

This completes the construction of $\phi_{\mathcal{F}, \mathcal{L}}$ in Eq. (22) and hence in Eq. (21).

It is clear that this correspondence sends the concatenation of rooted forests to the link of Motzkin paths and sends the grafting operator of rooted forests to the raising operator of peak-free Motzkin paths. Since $\left(\phi_{\mathcal{F}, \mathcal{L}} \circ \phi_{\mathcal{L}, \mathcal{F}}\right)\left(\bullet^{x} \bullet\right)=\bullet^{x} \bullet$ for $x \in X$, by the freeness of $\mathcal{L}(X, \Omega)$, we have $\phi_{\mathcal{F}, \mathcal{L}} \circ \phi_{\mathcal{L}, \mathcal{F}}=\mathrm{i} d_{\mathcal{L}(X, \Omega)}$, as needed.

(b) The proof follows in the same way as the proof of Corollary 3.7.

\section{Some natural bijections}

As noted in Example 1.3, well-known algebras with operators such as differential algebras, difference algebras and Rota-Baxter algebras are operated algebras with additional conditions on their operators. Thus the free objects in these categories are quotients of free operated algebras. In this context, results in previous sections show that free objects in these categories are quotients of the operated algebras of Motzkin paths or planar rooted forests. For some of these categories it is possible to find a canonical subset of the Motzkin paths or planar rooted forests that projects to a basis in these quotients. This is the case for Rota-Baxter algebras. It was shown in $[2,16]$ that free Rota-Baxter algebras on a set can be constructed from a subset of planar rooted forests with decorations on the angles. In Section 6, we will give similar constructions in terms of Motzkin paths and leaf decorated trees, as well as in terms of bracketed words which relates to the construction in [15]. For this purpose, we now put together bijections among these combinatorial objects. 


\subsection{Angularly decorated forests}

For later references, we review the concept of angularly decorated planar rooted forests. See [16] for further details.

Let $X$ be a non-empty set. Let $F \in \mathcal{F}$ with $\ell=\ell(F)$ leaves. Let $X^{F}$ denote the set of pairs $(F ; \vec{x})$ where $\vec{x}$ is in $X^{(\ell(F)-1)}$ with the convention that $X^{\bullet}=\{(\bullet ; 1)\}$. Let $X^{\mathcal{F}}=\bigcup^{\bullet}{ }_{F \in \mathcal{F}} X^{F}$. We call $(F ; \vec{x})$ an angularly decorated forest since it can be identified with the forest $F$ together with an ordered decoration by $\vec{x}$ on the angles of $F$. For example,

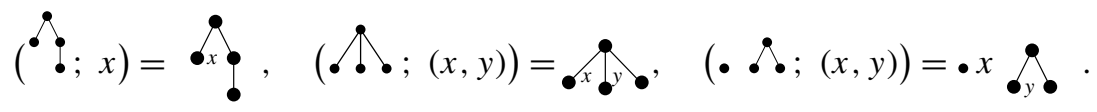

- $x$ ○ is denoted by $\bullet \sqcup_{x}$ ○ in [16].

Let $(F ; \vec{x}) \in X^{F}$. Let $F=T_{1} \cdots T_{b}$ be the decomposition of $F$ into trees. We consider the corresponding decomposition of the decorated forest. If $b=1$, then $F$ is a tree and $(F ; \vec{x})$ has no further decompositions. If $b>1$, denote $\ell_{i}=\ell\left(T_{i}\right)$, $1 \leq i \leq b$. Then

$$
\begin{aligned}
& \left(T_{1} ;\left(x_{1}, \cdots, x_{\ell_{1}-1}\right)\right),\left(T_{2} ;\left(x_{\ell_{1}+1}, \cdots, x_{\ell_{1}+\ell_{2}-1}\right)\right), \cdots, \\
& \left(T_{b} ;\left(x_{\ell_{1}+\cdots+\ell_{b-1}+1}, \cdots, x_{\ell_{1}+\cdots+\ell_{b}}\right)\right)
\end{aligned}
$$

are well-defined angularly decorated trees when $\ell\left(T_{i}\right)>1$. If $\ell\left(T_{i}\right)=1$, then $x_{\ell_{i-1}+\ell_{i}-1}=x_{\ell_{i-1}}$ and we use the convention $\left(T_{i} ; x_{\ell_{i-1}+\ell_{i}-1}\right)=\left(T_{i} ; \mathbf{1}\right)$. Thus we have,

$$
\begin{aligned}
\left(F ;\left(x_{1}, \cdots, x_{\ell-1}\right)\right)= & \left(T_{1} ;\left(x_{1}, \cdots, x_{\ell_{1}-1}\right)\right) x_{\ell_{1}}\left(T_{2} ;\left(x_{\ell_{1}+1}, \cdots, x_{\ell_{1}+\ell_{2}-1}\right)\right) x_{\ell_{1}+\ell_{2}} \\
& \cdots x_{\ell_{1}+\cdots+\ell_{b-1}}\left(T_{b} ;\left(x_{\ell_{1}+\cdots+\ell_{b-1}+1}, \cdots, x_{\ell_{1}+\cdots+\ell_{b}}\right)\right) .
\end{aligned}
$$

We call this the standard decomposition of $(F ; \vec{x})$ and abbreviate it as

$$
(F ; \vec{x})=\left(T_{1} ; \vec{x}_{1}\right) x_{i_{1}}\left(T_{2} ; \vec{x}_{2}\right) x_{i_{2}} \cdots x_{i_{b-1}}\left(T_{b} ; \vec{x}_{b}\right)=D_{1} x_{i_{1}} D_{2} x_{i_{2}} \cdots x_{i_{b-1}} D_{b}
$$

where $D_{i}=\left(T_{i} ; \vec{x}_{i}\right), 1 \leq i \leq b$. For example,

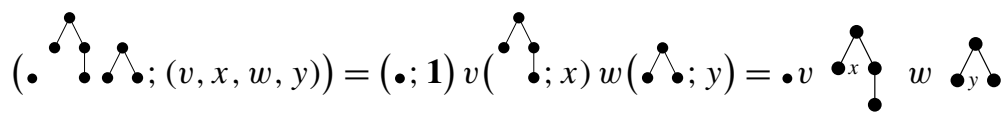

We note that even though $X^{\mathcal{F}}$ is not closed under concatenation of forests, it is closed under the grafting operator and $\lfloor(F ; \vec{x})\rfloor=(\lfloor F\rfloor ; \vec{x})$.

\subsection{The bijections}

We list below all the objects that we have encountered so far in order to study their relations in Theorem 5.1. Let $X$ be a non-empty set and let $\Omega$ be a singleton. Thus we will drop $\Omega$ in the following notations. 
(a) $\mathcal{M}(X)$ is the operated monoid of unitary bracketed words on the alphabet set $X$, defined in Eq. (11);

(b) $\mathcal{S}(X)$ is the operated semigroup of nonunitary bracketed words on the alphabet set $X$, defined in Eq. (12);

(c) $\mathcal{R}(X)$ is the set of Rota-Baxter bracketed words[15, 29] on the alphabet set $X$. Such a word is defined to be a word in $\mathcal{M}(X)$ that does not contain $\rfloor\lfloor$ as in $\lfloor x\rfloor\lfloor y\rfloor$;

(d) $\mathcal{P}(X)$ is the set of Motzkin paths with level steps decorated by $X$, considered in Corollary 2.2;

(e) $\mathcal{L}(X)$ is the set of peak-free Motzkin paths with level steps decorated by $X$, considered in Corollary 2.2;

(f) $\mathcal{V}(X)$ is the set of valley-free Motzkin paths with level steps decorated by $X$, consisting of Motzkin paths in $\mathcal{P}(X)$ with no down step followed immediately by an up step;

(g) $\mathcal{F}(X)$ is the set of planar rooted forests with leaves decorated by $X$, defined in Eq. (19);

(h) Define $\mathcal{F}_{\ell}(X)$ to be the subset of $\mathcal{F}(X)$ consisting of leaf decorated forests that do not have a vertex with adjacent non-leaf branches. Such a forest is called leafspaced. For example, the tree

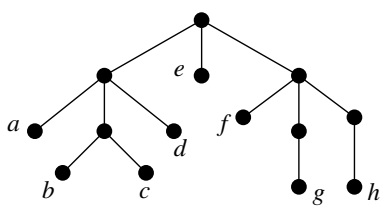

is not leaf-spaced since the two right most branches, with leaves decorated by $g$ and $h$, are not separated by a leaf branch. But the tree

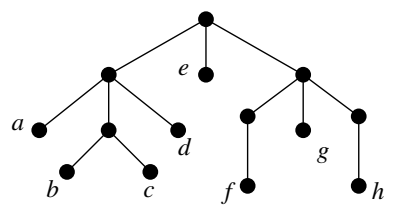

is leaf-spaced;

(i) $X^{\mathcal{F}}$ is the set of planar rooted tree with angles decorated by $X$, defined in Section 5.1;

(j) $X_{0}^{\mathcal{F}}$ be the subset of $X^{\mathcal{F}}$ consisting of ladder-free forests, namely those forests that do not have a ladder tree, the latter being defined to be a subtree $\neq \bullet$ with only one leaf. Equivalently, a ladder-free forest is a forest $\neq \bullet$ that does not have a subtree . For example, 6 is ladder-free, but is not ladder-free because of its right branch. 
Theorem 5.1 There are maps among the sets in the above list that fit into the following commutative diagram of bijection and inclusions.

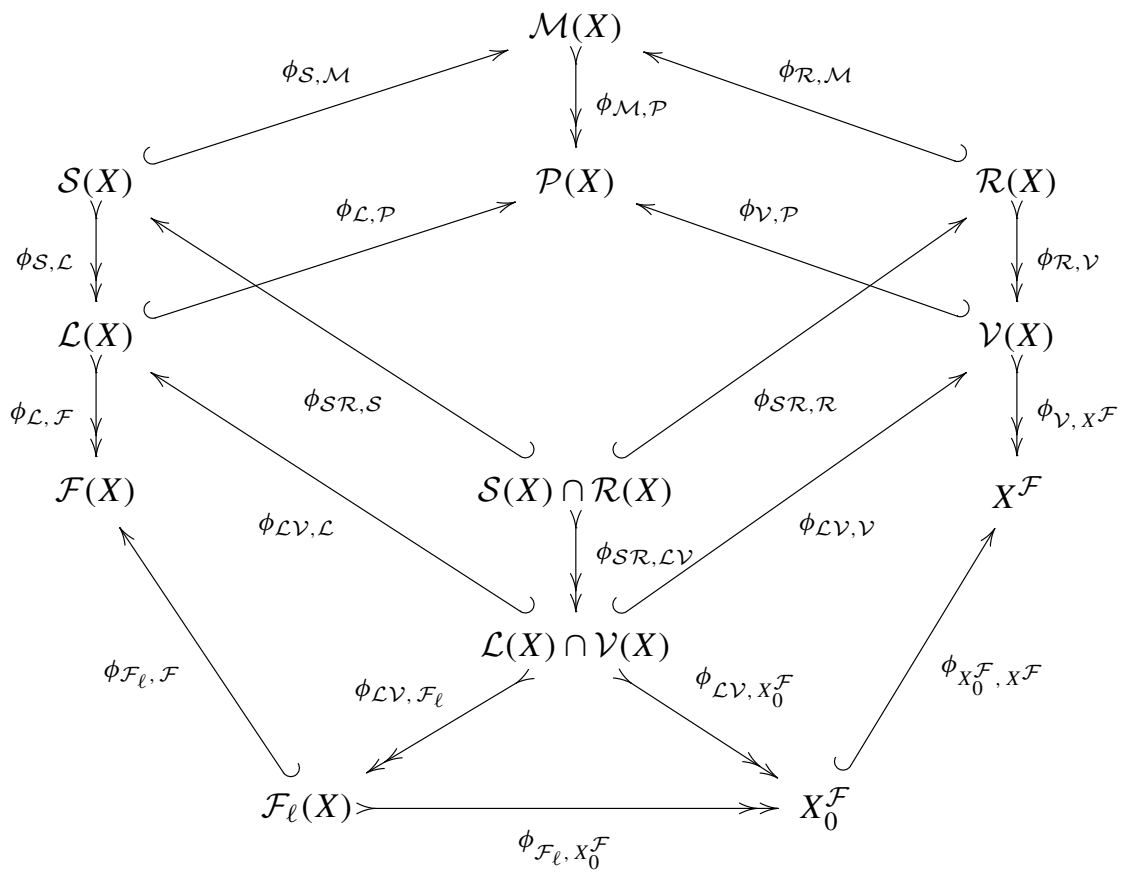

The maps will be recalled or defined in the proof. Each of the maps is compatible with the products, whenever defined, and is compatible with the distinguished operators.

Proof All the inclusions are clear from the definition of the sets. So we only need to verify the claimed properties for the bijective maps.

The isomorphism $\phi_{\mathcal{M}, \mathcal{P}}$ is obtained in Eq. (16). The isomorphism $\phi_{\mathcal{S}, \mathcal{L}}$ is the restriction of $\phi_{\mathcal{M}, \mathcal{P}}$. See Theorem 3.4.(c) and its proof. The isomorphism $\phi_{\mathcal{L}, \mathcal{F}}$ is defined in Eq. (20) whose inverse is $\phi_{\mathcal{F}, \mathcal{L}}$ in Eq. (21).

$\phi_{\mathcal{R}, \mathcal{V}}$ is the restriction of the operated monoid isomorphism $\phi_{\mathcal{M}, \mathcal{P}}$ to $\mathcal{R}(X)$. Its bijectivity follows from Theorem 3.4 and Remark 3.5.

$\phi_{\mathcal{S}}, \mathcal{L} \mathcal{V}$ is defined to be the restriction of $\phi_{\mathcal{M}, \mathcal{P}}$. Hence its bijectivity follows

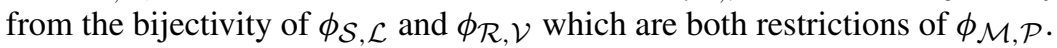

$\phi_{\mathcal{L} V}, \mathcal{F}_{\ell}$ is defined to be the restriction of the bijective map $\phi_{\mathcal{L}, \mathcal{F}}$ to $\mathcal{L}(X) \cap \mathcal{V}(X)$. By the explicit description of $\phi_{\mathcal{F}, \mathcal{L}}=\phi_{\mathcal{L}, \mathcal{F}}^{-1}$ in the proof of Theorem 4.2, a Motzkin path $\mathfrak{m} \in \mathcal{L}(X)$ has a peak if and only if the corresponding leaf decorated rooted forest $\phi_{\mathcal{L}, \mathcal{F}}(\mathfrak{m})=\phi_{\mathcal{L}, \mathcal{F}}(\mathfrak{m}) \in \mathcal{F}(X)$ has a vertex with two adjacent non-leaf branches. The bijectivity of $\phi_{\mathcal{L} \mathcal{V}, \mathcal{F}_{\ell}}$ follows.

We will define below a bijective map

$$
\phi_{\mathcal{V}, X} \mathcal{F}: \mathcal{V}(X) \rightarrow X^{\mathcal{F}}
$$


that restricts to a bijective map

$$
\phi_{\mathcal{L} \mathcal{V}, X_{0}^{\mathcal{F}}}: \mathcal{L}(X) \cap \mathcal{V}(X) \rightarrow X_{0}^{\mathcal{F}}
$$

and is compatible with the distinguished operators. Then by composition, we obtain a bijective map

$$
\phi_{\mathcal{F}_{\ell}, X_{0}^{\mathcal{F}}}=\phi_{\mathcal{L} \mathcal{V}, X_{0}^{\mathcal{F}}}^{\mathcal{O}} \circ \phi_{\mathcal{L} \mathcal{V}, \mathcal{F}_{\ell}}^{-1}: \mathcal{F}_{\ell}(X) \rightarrow \mathcal{L}(X) \cap \mathcal{V}(X) \rightarrow X_{0}^{\mathcal{F}}
$$

that is compatible with the distinguished operators.

Thus it remains to construct a bijective map

$$
\phi_{\mathcal{V}, X^{\mathcal{F}}}: \mathcal{V}(X) \rightarrow X^{\mathcal{F}}
$$

with the prescribed properties above. For this we give another canonical decomposition of a decorated Motzkin path $\bullet \neq \mathfrak{m} \in \mathcal{V}(X)$. Let

$$
\mathfrak{m}=\mathfrak{m}_{1} \circ \cdots \circ \mathfrak{m}_{p}
$$

be the decomposition of $\mathfrak{m} \neq \bullet$ into indecomposable decorated Motzkin paths $\mathfrak{m}_{i} \neq$ $\bullet, 1 \leq i \leq p$. Since $\mathfrak{m}$ is valley free, there are no consecutive $\mathfrak{m}_{i}$ and $\mathfrak{m}_{i+1}$ that have height greater or equal to 1 . Then add a path factor $\bullet$ to the beginning and the end of $\mathfrak{m}$, and insert a $\bullet$ between any two consecutive $\mathfrak{m}_{i}$ and $\mathfrak{m}_{i+1}$ of height zero. That is, rewrite $\mathfrak{m}_{i} \circ \mathfrak{m}_{i+1}=\bullet^{x} \bullet \circ \bullet^{y} \bullet$ as $\mathfrak{m}_{i} \circ \bullet \circ \mathfrak{m}_{i+1}$. Since $\bullet$ is the identity element of the product $\circ$, this rewriting does not change $\mathfrak{m}$. In this way Eq. (31) is uniquely rewritten as

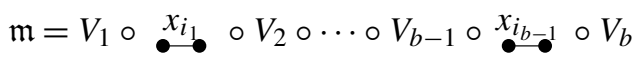

where each $V_{j}, 1 \leq j \leq b$, is either $\bullet$ or an indecomposable valley-free Motzkin path of height at least one. Call this the standard decomposition of $\mathfrak{m}$. As an example we have the following decomposition and standard decomposition of a Motzkin path.

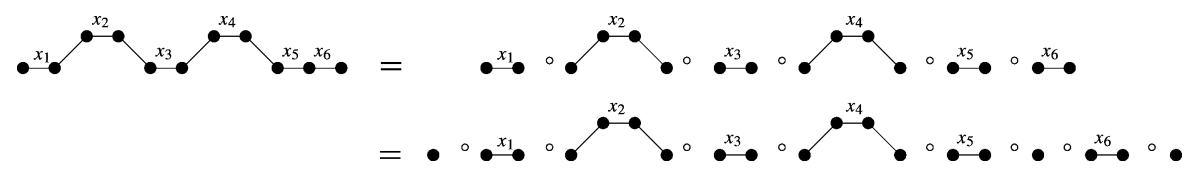

Note that an indecomposable valley-free Motzkin path $V_{j}$ of height $k \geq 1$ is of the form $/ \bar{V}_{j} \backslash$ for another Motzkin path $\bar{V}_{j}$ of height $k-1$. We then use induction on $k$ to define

$$
\phi_{\mathcal{V}, X^{\mathcal{F}}}(\mathfrak{m})= \begin{cases}(\bullet, \mathbf{1}), & \mathfrak{m}=\bullet, \\ \phi_{\mathcal{V}, X^{\mathcal{F}}}\left(V_{1}\right) x_{i_{1}} \phi_{\mathcal{V}, X^{\mathcal{F}}}\left(V_{2}\right) \cdots \phi_{\mathcal{V}, X^{\mathcal{F}}}\left(V_{b-1}\right) x_{i_{b-1}} \phi_{\mathcal{V}, X^{\mathcal{F}}}\left(V_{b}\right), \mathfrak{m} \neq \bullet\end{cases}
$$

with

$$
\phi_{\mathcal{V}, X^{\mathcal{F}}}\left(V_{j}\right)= \begin{cases}(\bullet, \mathbf{1}), & V_{j}=\bullet \\ \left\lfloor\phi_{\mathcal{V}, X} \mathcal{F}\left(\bar{V}_{j}\right)\right\rfloor, & V_{j}=/ \bar{V}_{j} \backslash\end{cases}
$$


Here the second case is well-defined by the induction hypothesis. It is clear that Eq. (33) gives the standard decomposition of a planar rooted forest in $X^{\mathcal{F}}$.

Conversely, for any given angularly decorated rooted forest $D \in X^{\mathcal{F}}$, let

$$
D=D_{1} x_{i_{1}} D_{2} \cdots D_{b-1} x_{i_{b-1}} D_{b}
$$

be its standard decomposition in Eq. (26). Then define

$$
\phi_{X}^{\mathcal{F}, \mathcal{V}}(D)=\phi_{X}^{\mathcal{F}, \mathcal{V}}\left(D_{1}\right) \circ \stackrel{x_{i_{1}}}{\bullet} \circ \phi_{X}^{\mathcal{F}, \mathcal{V}}\left(D_{2}\right) \circ \cdots \circ \stackrel{x_{i_{b-1}} \circ \phi_{X} \mathcal{F}, \mathcal{V}}{\bullet}\left(D_{b}\right)
$$

where on the right hand side

$$
\phi_{X^{\mathcal{F}}, \mathcal{V}}\left(D_{j}\right)= \begin{cases}\bullet, & D_{j}=(\bullet ; \mathbf{1}), \\ / \phi_{X^{\mathcal{F}}, \mathcal{V}}\left(\bar{D}_{j}\right) \backslash, & D_{j}=\left\lfloor\bar{D}_{j}\right\rfloor .\end{cases}
$$

Then clearly $\phi_{X} \mathcal{F}, \mathcal{V}$ is the inverse of $\phi_{\mathcal{V}, X} \mathcal{F}$.

Note that

$$
\phi_{X}^{\mathcal{F}, \mathcal{V}}(\bullet)=\phi_{X} \mathcal{F}, \mathcal{V}(\lfloor\bullet\rfloor)=/ \phi_{X^{\mathcal{F}}, \mathcal{V}}(\bullet) \backslash=\bullet
$$

Thus if $D \in X^{\mathcal{F}}$ does not have a subtree then $\phi_{X^{\mathcal{F}}, \mathcal{V}}(D)$ does not have a subpath , namely $\phi_{X} \mathcal{F}, \mathcal{V}(D)$ is peak free. This shows that $\phi_{X} \mathcal{F}, \mathcal{V}$ restricts to a bijection $\phi_{X_{0}^{\mathcal{F}}, \mathcal{L} \mathcal{V}}$ in Eq. (29). This completes the proof of the theorem.

Remark 5.2 (A combinatorial description of $\phi_{X} \mathcal{F}, \mathcal{V}$.) Let $D=(T ; \vec{x}$ ) with $T$ a planar rooted tree and $\vec{x}$ the vector of angular decorations on $T$. We first list the edges of $T$ in biorder. The edge biorder list of $T$ is defined as follows.

(a) If $T$ has only one vertex, then there is nothing in the edge biorder list of $T$;

(b) If $T$ has more than one vertices, then the root vertex of $T$ has subtrees $T_{1}, \cdots, T_{k}$, $k \geq 1$, listed from left to right. Then the edge biorder list of $T$ is

- $U$, followed by the edge biorder list of $T_{1}$, followed by $D$,

- $\cdots$,

- $U$, followed by the edge biorder list of $T_{k}$, followed by $D$.

Modifying the "worm" illustration of the vertex preorder in [42, Figure 5-14], imagine that a worm begins just left of the root of the tree and crawls counterclockwise along the outside of the tree until it returns to the starting point. As it crawls along, for each of the edges it passes, it records a / if it is crawling away from the root and records a $\backslash$ if it is crawling toward the root. Note that this way, each edge is recorded twice, the first by a / and the second time by a $\backslash$. For example, the edge biorder list of the angular decorated tree

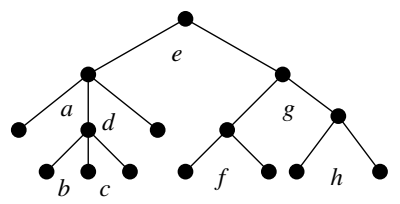


(ignoring the decorations for now) is

\section{$/ / / / / \backslash / \backslash / / / / / \backslash \backslash / / / \backslash \backslash$}

Regarding an edge biorder list as a word $\mathfrak{w}$ with the alphabet set $\{/, \backslash\}$, counting from the left of $\mathfrak{w}$, the number of occurrences of / (resp. $\backslash$ ) is the number of edges that are encountered for the first (resp. the second) time. Thus $\mathfrak{w}$ is a Motzkin word by its definition (Definition 3.1). In fact, it is a Dyck word in the sense that it codes a Dyck path.

Next we deduce a Motzkin word decorated by $L$ from this edge biorder list by replacing each occurrence of $\backslash /$ by an $L$. For example, the above edge biorder list is deduced to

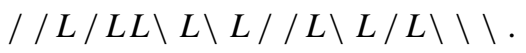

Note that each time the "worm" passes an angles of the tree it records a pair \/ in the edge biorder list and hence an $L$ in the corresponding Motzkin word. So the number of angles of the tree $T$ equals the number of $L$-letters in the Motzkin path. Thus we can use the entries of $\vec{x}$ in $D=(T ; \vec{x})$ to decorate and replace the $L$-letters, giving rise to an $X$-decorated Motzkin word. For our example above, we have

$$
/ / a / b c \backslash d \backslash e / / f \backslash g / h \backslash \backslash \backslash
$$

and hence the Motzkin path

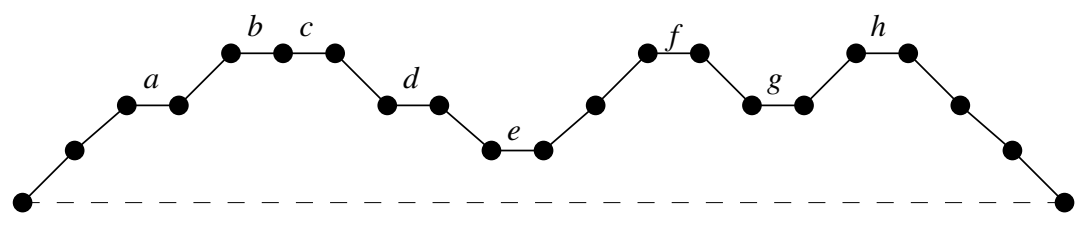

\section{Constructions of free Rota-Baxter algebras}

In [16] (see also [2]), free Rota-Baxter algebras on a set $X$ is constructed using angularly decorated planar forests. We first recall this construction and then show how, through the natural bijections in Theorem 5.1, we transport this Rota-Baxter algebra structure on angularly decorated forests to such a structure on Motzkin paths, leaf decorated planar forests and bracketed words.

\subsection{Review of the Rota-Baxter algebra structure on angularly decorated forests}

On the set of angularly decorated forests $X^{\mathcal{F}}$ defined in Section 5.1, define the free k-module $\mathbf{k} X^{\mathcal{F}}$ (denoted by $\amalg^{\mathrm{NC}}(X)$ in [16]). Note that if $D=(F ; \vec{x}) \in X^{\mathcal{F}}$ is a tree (that is, if $F$ is a tree), then since $F$ is either $\bullet$ or $\lfloor\bar{F}\rfloor$ for $\bar{F} \in \mathcal{F}$, we have either $D=(\bullet ; \mathbf{1})$ or $D=\lfloor\bar{D}\rfloor$ where $\bar{D}=(\bar{F} ; \vec{x})$. The depth filtration on $\mathcal{F}$ in Eq. (18) induces a depth filtration on $X^{\mathcal{F}}$. In [16], we use this filtration to define a multiplication $\bar{\diamond}$ on $\mathbf{k} X^{\mathcal{F}}$ that is characterized by the following properties. 
(a) $(\bullet ; \mathbf{1})$ is the multiplication identity;

(b) If $D$ and $D^{\prime}$ are angularly decorated trees not equal to $\bullet$, so $D=\lfloor\bar{D}\rfloor, D^{\prime}=\left\lfloor\bar{D}^{\prime}\right\rfloor$ for $\bar{D}, \bar{D}^{\prime} \in X^{\mathcal{F}}$, then

$$
D \bar{\diamond} D^{\prime}=\left\lfloor\bar{D} \bar{\diamond} D^{\prime}\right\rfloor+\left\lfloor D \bar{\diamond} \bar{D}^{\prime}\right\rfloor+\lambda\left\lfloor\bar{D} \bar{\diamond} \bar{D}^{\prime}\right\rfloor .
$$

(c) If $D=D_{1} x_{i_{1}} \cdots x_{i_{b-1}} D_{b}$ and $D^{\prime}=D_{1}^{\prime} x_{i_{1}^{\prime}}^{\prime} \cdots x_{i_{b^{\prime}-1}^{\prime}}^{\prime} D_{b^{\prime}}^{\prime}$ are the standard decomposition of the angularly decorated forests $D$ and $D^{\prime}$ in Eq. (26), then

$$
D \bar{\diamond} D^{\prime}=D_{1} x_{i_{1}} \cdots D_{b-1} x_{i_{b-1}}\left(D_{b} \bar{\diamond} D_{1}^{\prime}\right) x_{i_{1}^{\prime}}^{\prime} D_{2}^{\prime} \cdots x_{i_{b^{\prime}-1}^{\prime}}^{\prime} D_{b^{\prime}}
$$

For example, applying Eq. (37) and Eq. (38) we have

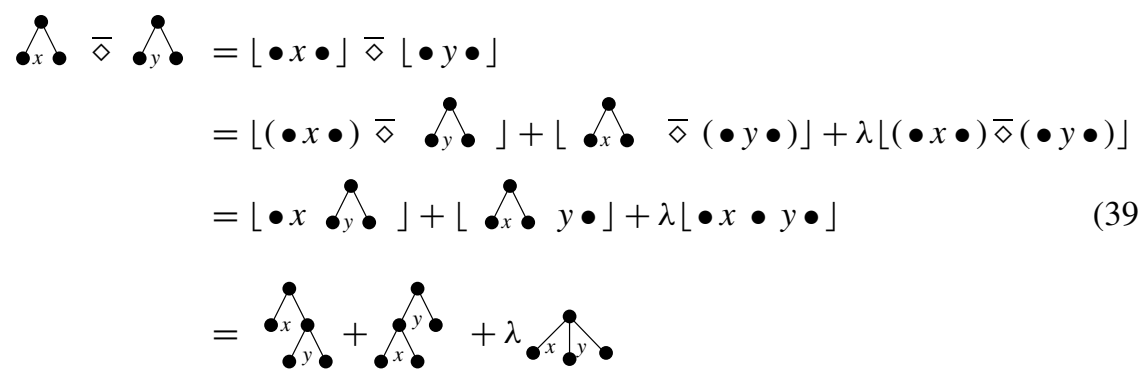

Extending the product $\bar{\diamond}$ bilinearly, we obtain a binary operation $\bar{\diamond}$ on $\mathbf{k} X^{\mathcal{F}}$. For $(F ; \vec{x}) \in X^{F}$, define

$$
P_{X}(F ; \vec{x})=\lfloor(F ; \vec{x})\rfloor=(\lfloor F\rfloor ; \vec{x}) \in X^{\lfloor F\rfloor},
$$

extending to a linear operator $P_{X}$ on $\mathbf{k} X^{\mathcal{F}}$. Let

$$
j_{X}: X \rightarrow \mathbf{k} X^{\mathcal{F}}
$$

be the map sending $x \in X$ to $(\bullet \bullet ; x)$. The following theorem is proved in [16].

Theorem 6.1 The quadruple $\left(\mathbf{k} X^{\mathcal{F}}, \bar{\diamond}, P_{X}, j_{X}\right)$ is the free Rota-Baxter algebra of weight $\lambda$ on the set $X$. More precisely, for any Rota-Baxter algebra $(R, P)$ and map $f: X \rightarrow R$, there is a unique Rota-Baxter algebra homomorphism $\bar{f}: \mathbf{k} X^{\mathcal{F}} \rightarrow R$ such that $f=\bar{f} \circ j_{X}$. Similarly, The quadruple $\left(\mathbf{k} X_{0}^{\mathcal{F}}, \bar{\diamond}, P_{X}, j_{X}\right)$ is the free nonunitary Rota-Baxter algebra of weight $\lambda$ on the set $X$. Here $X_{0}^{\mathcal{F}}$ is the set of ladder-free angularly decorated forests in Theorem 5.1.

\subsection{Rota-Baxter algebra structure on Motzkin paths}

We now transport the Rota-Baxter algebra structure from $\mathbf{k} X^{\mathcal{F}}$ to $\mathbf{k} \mathcal{V}(X)$ through the bijection $\phi_{X}{ }^{\mathcal{F}} \mathcal{V}$ in Eq. (35) and its inverse $\phi_{\mathcal{V}, X^{\mathcal{F}}}$ in Eq. (33). Note that an indecomposable $X$-decorated Motzkin path is either $\bullet$ or $\bullet \bullet$ or $/ \overline{\mathfrak{m}} \backslash$ for another $X$-decorated Motzkin path $\overline{\mathfrak{m}}$. 
Theorem 6.2 The bijection $\phi_{X} \mathcal{F}, \mathcal{V}: X^{\mathcal{F}} \rightarrow \mathcal{V}$ extends to an isomorphism

$$
\phi_{X} \mathcal{F}, \mathcal{V}:\left(\mathbf{k} X^{\mathcal{F}}, \bar{\diamond}, P_{X}\right) \rightarrow\left(\mathbf{k} \mathcal{V}, \diamond_{v},\lfloor\rfloor\right)
$$

of Rota-Baxter algebras where the multiplication $\diamond_{v}$ on $\mathbf{k} \mathcal{V}$ is defined recursively with respect to the height of Motzkin paths and is characterized by the following properties.

(a) The trivial path $\bullet$ is the multiplication identity;

(b) If $\mathfrak{m}$ and $\mathfrak{m}^{\prime}$ are indecomposable $X$-decorated Motzkin paths not equal to $\bullet$, then

$$
\mathfrak{m} \diamond_{v} \mathfrak{m}^{\prime}= \begin{cases}\mathfrak{m} \circ \mathfrak{m}^{\prime}, & \mathfrak{m}=\bullet^{x} \bullet \text { or } \mathfrak{m}^{\prime}=\bullet^{x^{\prime}} \bullet \\ / \overline{\mathfrak{m}} \diamond_{v} \mathfrak{m}^{\prime} \backslash+/ \mathfrak{m} \diamond \overline{\mathfrak{m}^{\prime}} \backslash+\lambda / \overline{\mathfrak{m}} \diamond_{v} \overline{\mathfrak{m}}^{\prime} \backslash, \mathfrak{m}=/ \overline{\mathfrak{m}} \backslash, \mathfrak{m}^{\prime}=/ \overline{\mathfrak{m}}^{\prime} \backslash ;\end{cases}
$$

(c) If $\mathfrak{m}=\mathfrak{m}_{1} \circ \cdots \circ \mathfrak{m}_{p}$ and $\mathfrak{m}^{\prime}=\mathfrak{m}_{1}^{\prime} \circ \cdots \circ \mathfrak{m}_{p^{\prime}}^{\prime}$ are the decompositions of $\mathfrak{m}, \mathfrak{m}^{\prime} \in$ $\mathcal{V}(X)$ into indecomposable paths, then

$$
\mathfrak{m} \diamond_{v} \mathfrak{m}^{\prime}=\mathfrak{m}_{1} \circ \cdots \circ\left(\mathfrak{m}_{p} \diamond_{v} \mathfrak{m}_{1}^{\prime}\right) \circ \cdots \circ \mathfrak{m}_{p^{\prime}}
$$

Further, the Rota-Baxter algebra isomorphism $\phi_{X} \mathcal{F}, \mathcal{V}$ restricts to an isomorphism

$$
\phi_{X_{0}^{\mathcal{F}}, \mathcal{L} \mathcal{V}}: \boldsymbol{k} X_{0}^{\mathcal{F}} \rightarrow \boldsymbol{k}(\mathcal{L} \cap \mathcal{V})
$$

of nonunitary Rota-Baxter algebras.

As an illustration, the example in Eq. (39) corresponds to

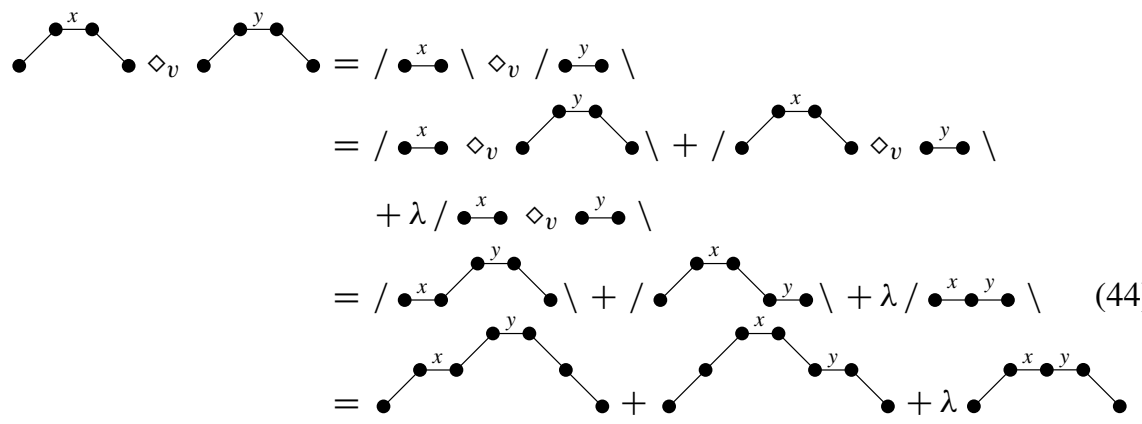

Proof We just need to show that under the bijection $\phi_{X} \mathcal{F}, \mathcal{V}$, the product $\triangleright$ on $\mathbf{k} X^{\mathcal{F}}$ characterized by (a) - (c) in $\S 6.1$ corresponds to the product $\diamond_{v}$ characterized by (a) - (c) in Theorem 6.2.

First of all, since $\phi_{X} \mathcal{F}, \mathcal{V}(\bullet)=\bullet \in \mathcal{V}(X), \bullet$ is the identity for the multiplication $\diamond_{v}$. Next let $\mathfrak{m}$ and $\mathfrak{m}^{\prime}$ be indecomposable $X$-decorated Motzkin paths that are not $\bullet$ 
Then either $\mathfrak{m}=\bullet^{x} \bullet$ or $\mathfrak{m}=/ \overline{\mathfrak{m}} \backslash$. Similarly for $\mathfrak{m}^{\prime}$. Then by Eq. (34), we have

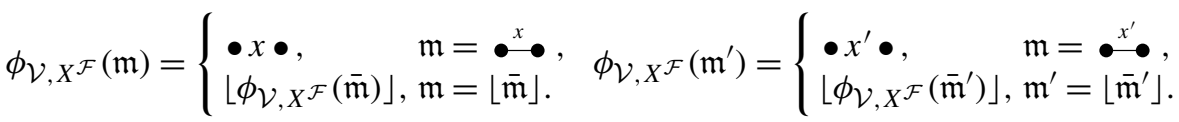

Denote

$$
D=\phi_{\mathcal{V}, X^{\mathcal{F}}}(\mathfrak{m}), D^{\prime}=\phi_{\mathcal{V}, X^{\mathcal{F}}}\left(\mathfrak{m}^{\prime}\right), \bar{D}=\phi_{\mathcal{V}, X^{\mathcal{F}}}(\overline{\mathfrak{m}}), \bar{D}^{\prime}=\phi_{\mathcal{V}, X^{\mathcal{F}}}\left(\overline{\mathfrak{m}}^{\prime}\right)
$$

It then follows from Eq. (37) and (38) that

$$
\begin{aligned}
& \phi_{\mathcal{V}, X} \mathcal{F}(\mathfrak{m}) \bar{\diamond} \phi_{\mathcal{V}, X} \mathcal{F}\left(\mathfrak{m}^{\prime}\right) \\
& = \begin{cases}\bullet x \bullet x^{\prime} \bullet, & \mathfrak{m}=\bullet^{x} \bullet, \mathfrak{m}^{\prime}=\bullet{ }^{x^{\prime}} \bullet, \\
\bullet x\left\lfloor\bar{D}^{\prime}\right\rfloor, & \mathfrak{m}=\bullet^{x} \bullet, \mathfrak{m}^{\prime}=/ \overline{\mathfrak{m}}^{\prime} \backslash, \\
\lfloor\bar{D}\rfloor x^{\prime} \bullet, & \mathfrak{m}=/ \overline{\mathfrak{m}} \backslash, \mathfrak{m}^{\prime}=\bullet{ }^{x^{\prime}} \bullet \\
\left\lfloor\bar{D} \bar{\diamond} D^{\prime}\right\rfloor+\left\lfloor D \bar{\diamond} \bar{D}^{\prime}\right\rfloor+\lambda\left\lfloor\bar{D} \bar{\diamond}^{\prime}\right\rfloor, & \mathfrak{m}=/ \overline{\mathfrak{m}} \backslash, \mathfrak{m}^{\prime}=/ \overline{\mathfrak{m}}^{\prime} \backslash .\end{cases}
\end{aligned}
$$

By definition, the product $\diamond_{v}$ on $\mathbf{k} \mathcal{V}$ is obtained from the product $\bar{\diamond}$ on $\mathbf{k} X^{\mathcal{F}}$ by

$$
\mathfrak{m} \diamond_{v} \mathfrak{m}^{\prime}=\phi_{X} \mathcal{F}, \mathcal{V}\left(\phi_{\mathcal{V}, X} \mathcal{F}(\mathfrak{m}) \bar{\diamond} \phi_{\mathcal{V}, X} \mathcal{F}\left(\mathfrak{m}^{\prime}\right)\right)
$$

Thus by Eq. (35) and (36) we have

$$
\mathfrak{m} \diamond_{v} \mathfrak{m}^{\prime}= \begin{cases}\bullet^{x} \bullet \circ \bullet^{x^{\prime}} \bullet=\mathfrak{m} \circ \mathfrak{m}^{\prime}, & \mathfrak{m}=\bullet^{x} \bullet, \mathfrak{m}^{\prime}=\bullet^{x^{\prime}} \bullet \\ \bullet^{x} \bullet \circ / \overline{\mathfrak{m}^{\prime}} \backslash=\mathfrak{m} \circ \mathfrak{m}^{\prime}, & \mathfrak{m}=\bullet^{x} \bullet, \mathfrak{m}^{\prime}=/ \overline{\mathfrak{m}}^{\prime} \backslash, \\ / \overline{\mathfrak{m}} \backslash \circ \bullet^{x^{\prime}} \bullet=\mathfrak{m} \circ \mathfrak{m}^{\prime}, & \mathfrak{m}=/ \overline{\mathfrak{m}} \backslash, \mathfrak{m}^{\prime}=\bullet^{x^{\prime}} \bullet \\ / \overline{\mathfrak{m}} \diamond_{v} \mathfrak{m}^{\prime} \backslash+/ \mathfrak{m} \diamond_{v} \overline{\mathfrak{m}^{\prime}} \backslash+\lambda / \overline{\mathfrak{m}} \diamond_{v} \overline{\mathfrak{m}}^{\prime} \backslash, \mathfrak{m}=/ \overline{\mathfrak{m}} \backslash, \mathfrak{m}^{\prime}=/ \overline{\mathfrak{m}}^{\prime} \backslash\end{cases}
$$

This is Eq. (42).

Let $\mathfrak{m}=\mathfrak{m}_{1} \circ \cdots \circ \mathfrak{m}_{p}$ and $\mathfrak{m}^{\prime}=\mathfrak{m}_{1}^{\prime} \circ \cdots \circ \mathfrak{m}_{p^{\prime}}^{\prime}$ be the decompositions of $\mathfrak{m}, \mathfrak{m}^{\prime} \in$ $\mathcal{V}(X)$ into indecomposable paths. Let $\mathfrak{m}=V_{1} \circ x_{i_{1}} \circ V_{2} \circ \cdots \circ x_{\bullet_{b-1}} \circ V_{b}$ and $\mathfrak{m}=V_{1}^{\prime} \circ{\stackrel{\bullet}{i_{b}^{\prime}}}_{\bullet}^{\prime} \circ V_{2}^{\prime} \circ \cdots \circ \stackrel{x_{b^{\prime}-1}^{\prime}}{\prime \prime} \circ V_{b^{\prime}}^{\prime}$ be their standard decompositions in Eq. (32). Then by Eq. (33), (35) and (38), we have

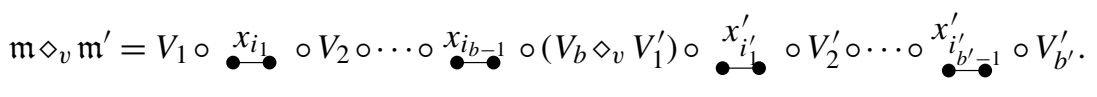

We check that no matter $\mathfrak{m}_{p}=\bullet \stackrel{x}{\bullet}$ or not and no matter $\mathfrak{m}_{1}^{\prime}=\bullet \stackrel{x^{\prime}}{\bullet}$ or not, Eq. (45) agrees with Eq. (43). This completes the proof that $\phi_{X^{\mathcal{F}}, \mathcal{V}}$ in Eq. (35) is a Rota-Baxter algebra isomorphism.

Since $\phi_{X^{\mathcal{F}}, \mathcal{V}}$ restricts to a bijection $\phi_{X_{0}^{\mathcal{F}}, \mathcal{L} \mathcal{V}}: X_{0}^{\mathcal{F}} \rightarrow \mathcal{L} \cap \mathcal{V}$ by Theorem 5.1 (see Eq. (29)), the second part of the theorem follows. 
Furthermore the map $j_{X}: X \rightarrow X^{\mathcal{F}}$ in Eq. (41) is translated to

$$
j_{X}: X \rightarrow \mathcal{P}(X), \quad j_{X}(x)=\bullet^{x} \bullet .
$$

Then by Theorem 6.1 and Theorem 6.2 we have

Corollary 6.3 The quadruple $\left(\mathbf{k} \mathcal{V}(X), \diamond_{v}, / \backslash, j_{X}\right)\left(\operatorname{resp} .\left(\mathbf{k}(\mathcal{V}(X) \cap \mathcal{L}(X)), \diamond_{v}, /\right.\right.$ $\left.\backslash, j_{X}\right)$ ) is the free Rota-Baxter algebra (resp. free nonunitary Rota-Baxter algebra) on $X$.

\subsection{Rota-Baxter algebra structure on bracketed words}

Through the natural bijection $\phi_{\mathcal{R}, \mathcal{V}}: \mathcal{R}(X) \rightarrow \mathcal{V}(X)$ in Theorem 5.1, the Rota-Baxter algebra structure on $\mathbf{k} \mathcal{V}(X)$ in Theorem 6.2 is transported to a Rota-Baxter algebra structure on $\mathbf{k} \mathcal{R}(X)$, giving another construction of the free Rota-Baxter algebra on $X$, in terms of bracketed words. See also $[15,29]$ for variations of this construction.

A bracketed word $W \in \mathcal{M}(X)$ is called indecomposable if either $W=\mathbf{1}$ or $W=x \in X$ or $W=\lfloor\bar{W}\rfloor$ where $\bar{W}$ is another bracketed word. Clearly, $W \in \mathcal{M}(X)$ is indecomposable if and only if the Motzkin path $\phi_{\mathcal{M}, \mathcal{P}}(W) \in \mathcal{P}(X)$ is indecomposable. It then follows that any bracketed word has a unique decomposition as a product of indecomposable bracketed words.

Since $\phi_{\mathcal{M}, \mathcal{P}}$ is an isomorphism of operated monoids, its restriction $\phi_{\mathcal{R}, \mathcal{V}}$ and its inverse $\phi \mathcal{V}, \mathcal{R}$ are compatible with the multiplications (link product on paths and concatenation product on words) and the distinguished operators (the raising and bracketing operators). Then we transport the product $\diamond_{v}$ on $\mathbf{k} X^{\mathcal{F}}$, characterized by (a)-(c) in Theorem 6.2, to a product $\diamond_{w}$ on $\mathbf{k} \mathcal{R}(X)$, characterized by the following properties.

(a) $\mathbf{1}$ is the multiplication identity.

(b) If $W$ and $W^{\prime}$ are indecomposable words in $\mathcal{R}(X)$ not equal to $\mathbf{1}$, then

$$
W \diamond_{w} W^{\prime}=\left\{\begin{array}{l}
W W^{\prime} \text { (word concatenation), } \quad W=x \in X \text { or } W^{\prime}=x^{\prime} \in X, \\
\left\lfloor\bar{W} \diamond_{w} W^{\prime}\right\rfloor+\left\lfloor W \diamond_{w} \bar{W}^{\prime}\right\rfloor+\lambda\left\lfloor\bar{W} \diamond_{w} \bar{W}^{\prime}\right\rfloor, \\
W=\lfloor\bar{W}\rfloor, W^{\prime}=\left\lfloor\bar{W}^{\prime}\right\rfloor .
\end{array}\right.
$$

(c) If $W=W_{1} \cdots W_{b}$ and $W^{\prime}=W_{1}^{\prime} \cdots W_{b^{\prime}}^{\prime}$ are the decompositions of $W, W^{\prime} \in \mathcal{R}(X)$ into indecomposable words, then

$$
W \diamond_{w} W^{\prime}=W_{1} \cdots\left(W_{b} \diamond_{w} W_{1}^{\prime}\right) \cdots W_{b^{\prime}}^{\prime}
$$

The example in Eq. (44) corresponds to

$$
\lfloor x\rfloor \diamond_{w}\lfloor y\rfloor=\left\lfloor x \diamond_{w}\lfloor y\rfloor\right\rfloor+\left\lfloor\lfloor x\rfloor \diamond_{w} y\right\rfloor+\lambda\left\lfloor x \diamond_{w} y\right\rfloor=\lfloor x\lfloor y\rfloor\rfloor+\lfloor\lfloor x\rfloor y\rfloor+\lambda\lfloor x y\rfloor
$$

Further the map $j_{X}: X \rightarrow \mathcal{R}(X)$ in Eq. (46) is transported to

$$
j_{X}: X \rightarrow \mathcal{S}(X) \cap \mathcal{R}(X) \subseteq \mathcal{R}(X), \quad j_{X}(x)=x .
$$

Then by Theorem 6.2 and Corollary 6.3 we have 


\section{Corollary 6.4}

(a) The bijection $\phi_{\mathcal{R}, \mathcal{V}}: \mathcal{R}(X) \rightarrow \mathcal{V}(X)$ extends to an isomorphism $\phi_{\mathcal{R}, \mathcal{V}}$ : $\left(\mathbf{k} \mathcal{R}(X), \diamond_{w},\lfloor\rfloor\right) \rightarrow\left(\mathbf{k} \mathcal{V}(X), \diamond_{v}, / \backslash\right)$ of Rota-Baxter algebras. This isomorphism restricts to an isomorphism $\phi_{\mathcal{S} \mathcal{R}, \mathcal{L} \mathcal{V}}:\left(\mathbf{k}(\mathcal{S}(X) \cap \mathcal{R}(X)), \diamond_{w},\lfloor\rfloor\right) \rightarrow$ $\left(\mathbf{k}(\mathcal{L}(X) \cap \mathcal{V}(X)), \diamond_{v}, / \backslash\right)$ of nonunitary Rota-Baxter algebras.

(b) The quadruple $\left.\left(\mathbf{k} \mathcal{R}(X), \diamond_{w}, \downarrow\right\rfloor, j_{X}\right)\left(\right.$ resp. $\left.\left(\mathbf{k}(\mathcal{S}(X) \cap \mathcal{R}(X)), \diamond_{w},\lfloor\rfloor, j_{X}\right)\right)$ is the free Rota-Baxter algebra (resp. free nonunitary Rota-Baxter algebra) on $X$.

\subsection{Rota-Baxter algebra structure on leaf decorated rooted forests}

We finish this paper by obtaining a free Rota-Baxter algebra structure on the free $\mathbf{k}$-module $\mathbf{k} \mathcal{F}_{\ell}(X)$ of leaf decorated rooted forests.

Through the bijection $\phi_{\mathcal{L} \mathcal{V}, \mathcal{F}_{\ell}}: \mathcal{L}(X) \cap \mathcal{V}(X) \rightarrow \mathcal{F}_{\ell}(X)$ in Theorem 5.1, the free nonunitary Rota-Baxter algebra on $\mathbf{k}(\mathcal{L}(X) \cap \mathcal{V}(X))$ in Corollary 6.3 gives us a free nonunitary Rota-Baxter algebra structure on $\mathbf{k} \mathcal{F}_{\ell}(X)$. Since $\phi_{\mathcal{L} \mathcal{V}, \mathcal{F}_{\ell}}$ sends the link product of paths to the concatenation of forests and sends the raising operator to the grafting operator, the two properties in Eq. (42) and Eq. (43) translate to the following properties characterizing the multiplication $\diamond_{\ell}$ on leaf-spaced leaf decorated forests.

(a) If $F$ and $F^{\prime}$ are leaf decorated trees, then

$$
F \diamond_{\ell} F^{\prime}= \begin{cases}F F^{\prime} \text { (concatenation of trees), } & F=\bullet_{x} \text { or } F^{\prime}=\bullet^{\prime}, \\ \left\lfloor\bar{F} \diamond_{\ell} F^{\prime}\right\rfloor+\left\lfloor F \diamond_{\ell} \bar{F}^{\prime}\right\rfloor+\lambda\left\lfloor\bar{F} \diamond_{\ell} \bar{F}^{\prime}\right\rfloor, & F=\lfloor\bar{F}\rfloor, F^{\prime}=\left\lfloor\bar{F}^{\prime}\right\rfloor .\end{cases}
$$

(b) If $F=F_{1} \cdots F_{b}$ and $F^{\prime}=F_{1}^{\prime} \cdots F_{b^{\prime}}^{\prime}$ are in $\mathcal{F}_{\ell}(X)$ with their corresponding decomposition into leaf decorated trees, then

$$
F \diamond_{\ell} F^{\prime}=F_{1} \cdots\left(F_{b} \diamond_{\ell} F_{1}^{\prime}\right) \cdots F_{b^{\prime}}^{\prime}
$$

The example in Eq. (44) translates to

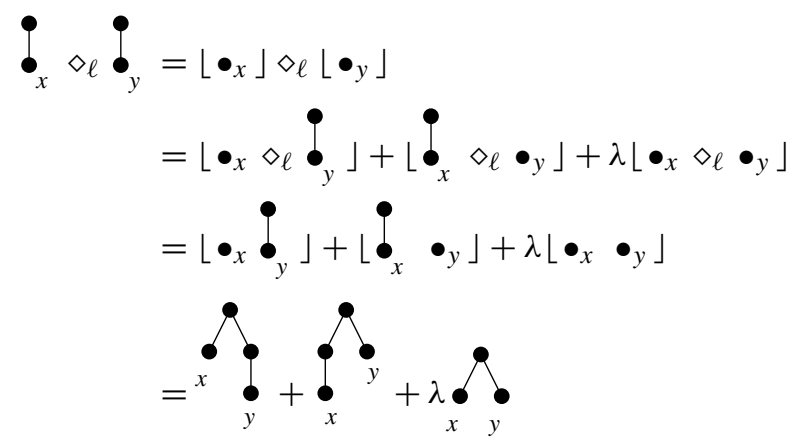

Further the map $j_{X}: X \rightarrow \mathcal{L}(X) \cap \mathcal{V}(X)$ in Eq. (46) is transported to

$$
j_{X}: X \rightarrow \mathcal{F}_{\ell}(X), \quad j_{X}(x)=\bullet_{x}, x \in X .
$$

Then by Theorem 6.2 and Corollary 6.3 we have 


\section{Corollary 6.5}

(a) The bijection $\phi_{\mathcal{L V}, \mathcal{F}_{\ell}}: \mathcal{L}(X) \cap \mathcal{V}(X) \rightarrow \mathcal{F}_{\ell}(X)$ extends to an isomorphism $\phi_{\mathcal{L} \mathcal{V}, \mathcal{F}_{\ell}}:\left(\mathbf{k}(\mathcal{L}(X) \cap \mathcal{V}(X)), \diamond_{v}, / \backslash\right) \rightarrow\left(\mathbf{k} \mathcal{F}_{\ell}(X), \diamond_{\ell},\lfloor\rfloor\right)$ of nonunitary RotaBaxter algebras.

(b) The quadruple $\left(\mathbf{k} \mathcal{F}_{\ell}(X), \diamond_{\ell},\lfloor\rfloor, j_{X}\right)$ is the free nonunitary Rota-Baxter algebra on $X$.

Acknowledgements The author thanks NSF grant DMS-0505643 for support and thanks the referee whose comments help to improve the presentation of the paper.

\section{References}

1. Aguiar, M.: On the associative analog of Lie bialgebras. J. Algebra 244, 492-532 (2001)

2. Aguiar, M., Moreira, W.: Combinatorics of the free Baxter algebra. Electron. J. Comb. 13, R17 (2006). arXiv:math.CO/0510169

3. Alonso, L.: Uniform generation of a Motzkin word. Theor. Comput. Sci. 134, 529-536 (1994)

4. Baxter, G.: An analytic problem whose solution follows from a simple algebraic identity. Pac. J. Math. 10, 731-742 (1960)

5. Benchekroun, S., Moszkowski, P.: A new bijection between ordered trees and legal bracketings. Eur. J. Comb. 17, 605-611 (1996)

6. Bertrand, T.: Solution d'un problème. C.R. Acad. Sci. Paris 105, 369 (1887)

7. Cartier, P.: On the structure of free Baxter algebras. Adv. Math. 9, 253-265 (1972)

8. Chen, W.Y.C., Shapiro, L.W., Yang, L.L.M.: Parity reversing involutions on plane trees and 2-Motzkin paths. Eur. J. Comb. 27, 283-289 (2006)

9. Cohn, R.M.: Difference Algebra. Interscience, New York (1965)

10. Connes, A., Kreimer, D.: Hopf algebras, renormalization and noncommutative geometry. Commun. Math. Phys. 199, 203-242 (1998)

11. Diestel, R.: Graph Theory, 3rd edn. Springer, Berlin (2005). Available on-line: http://www.math.unihamburg.de/home/diestel/books/graph.theory/download.html

12. Deutsch, E., Shapiro, L.W.: A bijection between ordered trees and 2-Motzkin paths and its many consequences. Discrete Math. 256, 655-670 (2002)

13. Donaghey, R., Shapiro, L.W.: Motzkin numbers. J. Comb. Theory Ser. A 23, 291-301 (1977)

14. Ebrahimi-Fard, K., Gracia-Bondia, J.M., Patras, F.: A Lie theoretic approach to renormalization. Commun. Math. Phys. 81, 61-75 (2007). arXiv:hep-th/0609035

15. Ebrahimi-Fard, K., Guo, L.: Rota-Baxter algebras and dendriform dialgebras. J. Pure Appl. Algebra (to appear). arXiv: math.RA/0503647

16. Ebrahimi-Fard, K., Guo, L.: Free Rota-Baxter algebras and rooted trees. J. Algebra Appl. (accepted). arXiv:math.RA/0510266

17. Ebrahimi-Fard, K., Guo, L., Kreimer, D.: Integrable renormalization II: the general case. Ann. Henri Poincare 6, 369-395 (2005)

18. Ebrahimi-Fard, K., Guo, L., Kreimer, D.: Spitzer's identity and the algebraic Birkhoff decomposition in pQFT. J. Phys. A: Math. Gen. 37, 11037-11052 (2004)

19. Ebrahimi-Fard, K., Guo, L., Manchon, D.: Birkhoff type decompositions and the Baker-CampbellHausdorff recursion. Commun. Math. Phys. 267, 821-845 (2006)

20. Engel, K.-J., Nagel, R.: One-Parameter Semigroups for Linear Evolution Equations. Graduate Texts in Mathematics, vol. 194. Springer, New York (2000)

21. Flajolet, P.: Mathematical methods in the analysis of algorithms and data structures. In: Trends in Theoretical Computer Science, Udine, 1984. Principles Comput. Science Ser., vol. 12, pp. 225-304. Computer Sci. Press, Rockville (1988)

22. Grillet, R.A.: Commutative Semigroups. Springer, Berlin (2006)

23. Grossman, R., Larson, R.G.: Hopf-algebraic structures of families of trees. J. Algebra 26, 184-210 (1989)

24. Guo, L.: Baxter algebras and the umbral calculus. Adv. Appl. Math. 27, 405-426 (2001) 
25. Guo, L.: Baxter algebras, Stirling numbers and partitions. J. Algebra Appl. 4, 153-164 (2005)

26. Guo, L., Keigher, W.: Baxter algebras and shuffle products. Adv. Math. 150, 117-149 (2000)

27. Guo, L., Keigher, W.: On free Baxter algebras: completions and the internal construction. Adv. Math. 151, 101-127 (2000)

28. Guo, L., Keigher, W.: On differential Rota-Baxter algebras. J. Pure Appl. Algebra (to appear). arXiv: math.RA/0703780

29. Guo, L., Yu Sit, W.: Enumenation of Rota-Baxter words. In: Proceedings ISSAC 2006, Genoa, Italy, pp. 124-131. ACM Press, New York (2006)

30. Guo, L., Zhang, B.: Renormalization of multiple zeta values. J. Algebra (to appear). arXiv:math.NT/0606076

31. Guo, L., Zhang, B.: Differential Birkhoff decomposition and renormalization of multiple zeta values. J. Number Theory (to appear)

32. Hofmann, K.H., Lawson, J.D., Vinberg, E.B.: Semigroups in Algebra, Geometry and Analysis. de Gruyter, Berlin (1995)

33. Kolchin, E.: Differential Algebra and Algebraic Groups. Academic Press, New York (1973)

34. Loday, J.-L., Ronco, M.: Trialgebras and families of polytopes. In: Homotopy Theory: Relations with Algebraic Geometry, Group Cohomology, and Algebraic K-theory. Contemporary Mathematics, vol. 346, pp. 369-398 (2004)

35. MacLane, S.: Categories for the Working Mathematician. Springer, New York (1971)

36. Rota, G.: Baxter algebras and combinatorial identities I. Bull. Am. Math. Soc. 5, 325-329 (1969)

37. Rota, G.: Baxter operators, an introduction. In: Kung, J.P.S. (ed.) Gian-Carlo Rota on Combinatorics, Introductory Papers and Commentaries, pp. 504-512. Birkhäuser, Boston (1995)

38. Sapounakis, A., Tsikouras, P.: On $k$-colored Motzkin words, J. Integer Seq. 7 (2004), Article 04.2.5

39. Shum, K.P., Guo, Y., Ito, M., Fong, Y. (eds.): Semigroups, the International Conference on Semigroups and its Related Topics. Springer, Berlin (1998)

40. Singer, M. (eds.): Talk at the Second International Workshop on Differential Algebra and Related Topics, Rutgers University, Newark, 12-13 April 2007

41. Singer, M., van der Put, M.: Galois Theory of Linear Differential Equations. Springer, Berlin (2003)

42. Stanley, R.R.: Enumerative Combinatorics, vol. 2. Cambridge University Press, Cambridge (1999)

43. Weisstein, E.W., Tree. From MathWorld. http://mathworld.wolfram.com/Tree.html 